\section{Cureus}

\title{
Cardio-Oncology: Cancer Therapy-related Cardiovascular Complications in a Molecular Targeted Era: New Concepts and Perspectives
}

David Hurtado-de-Mendoza ${ }^{1}$, Arturo Loaiza-Bonilla ${ }^{2}$, Paula A. Bonilla-Reyes ${ }^{3}$, Gabriel Tinoco ${ }^{4}$ , Rodrigo Alcorta ${ }^{5}$

1. University of Miami Miller School of Medicine, University of Miami Miller School of Medicine/Jackson Memorial Hospital, Miami, USA 2. Medicine, Hematology and Oncology, Cancer Treatment Centers of America 3. Facultad de Medicina, Pontificia Universidad Javeriana 4. Department of Internal Medicine, The Ohio State University College of Medicine 5. Facultad de Medicina, Universidad Peruana Cayetano Heredia

$\square$ Corresponding author: David Hurtado-de-Mendoza, davidmarshall2016@gmail.com Disclosures can be found in Additional Information at the end of the article

\section{Abstract}

Cardio-oncology is a medical discipline that identifies, prevents, and treats the cardiovascular complications related to cancer therapy. Due to the remarkable proliferation of new cancer therapies causing cardiovascular complications, such as hypertension, heart failure, vascular complications, and cardiac arrhythmia, we provide an extensive, comprehensive revision of the most up-to-date scientific information available on the cardiovascular complications associated with the use of newer, novel chemotherapeutic agents, including their reported incidence, suggested pathophysiology, clinical manifestations, potential treatment, and prevention. The authors consider this topic to be relevant for the clinicians since cardiovascular complications associated with the administration of recently approved drugs are relatively underappreciated.

The purpose of this article is to provide a state-of-the-art review of cardiovascular complications associated with the use of newer, novel chemotherapeutic agents and targeted therapies, including their reported incidence, suggested pathophysiology, clinical manifestations, potential treatment, and prevention.

Ongoing efforts are needed to provide a better understanding of the frequency, mechanisms of disease, prevention, and treatment of cardiovascular complications induced by the newer, novel chemotherapeutic agents. Development of a cardio-oncology discipline is warranted in order to promote task forces aimed at the creation of oncology patient-centered guidelines for the detection, prevention, and treatment of potential cardiovascular side effects associated with newer cancer therapies.

Received 09/14/2016

Review began $02 / 08 / 2017$ Review ended 05/09/2017 Published 05/18/2017

\section{(C) Copyright 2017}

Hurtado-de-Mendoza et al. This is an open access article distributed under the terms of the Creative Commons Attribution License CC-BY 3.0., which permits unrestricted use, distribution, and reproduction in any medium, provided the original author and source are credited.
Categories: Internal Medicine, Cardiology, Oncology

Keywords: cancer treatment, cardiac dysfunction, cardiotoxicity, cardio-oncology, cardiovascular events

\section{Introduction And Background}

Cancer is one of the top leading causes of death in the world. As a result of improved survival with novel cancer therapies, cardiovascular disease is a prominent cause of death in many cancer survivors, with cardiotoxicity being a serious side effect of chemotherapy and radiation therapy. The cardiotoxicity profile of the various chemotherapeutic agents, mechanisms of disease and potential approaches to prevention of cardiovascular disease differ substantially. While the cardiotoxic effects of time-honored chemotherapeutic agents, such as anthracyclines and alkylating agents, are well recognized and extensively studied, the cardiovascular complications associated with the administration of recently approved drugs are relatively underappreciated.

\section{How to cite this article}

Hurtado-de-Mendoza D, Loaiza-Bonilla A, Bonilla-Reyes P A, et al. (May 18, 2017) Cardio-Oncology: Cancer Therapy-related Cardiovascular Complications in a Molecular Targeted Era: New Concepts and Perspectives. Cureus 9(5): e1258. DOI 10.7759/cureus.1258 


\section{Cureus}

The purpose of this article is to provide a state-of-the-art review of cardiovascular complications (i.e., hypertension, myocardial ischemia and infarction (MI), heart failure, thromboembolism, QT prolongation, and arrhythmias) associated with the use of newer, novel chemotherapeutic agents and targeted therapies, including their reported incidence, suggested pathophysiology, clinical manifestations, potential treatment, and prevention.

\section{Review}

\section{Small molecule tyrosine kinase inhibitors}

The human genome contains about 90 tyrosine kinase and 43 tyrosine kinase (TK)-like genes whose expression translates into two important groups: transmembrane receptor and intracellular nonreceptor tyrosine kinases. The overexpression and/or mutation of tyrosine kinase signaling proteins have been shown to cause abnormal cell proliferation and differentiation, angiogenesis, and inhibition of apoptosis [1-2].

Tyrosine kinase inhibitors (TKIs) are small molecules that inhibit phosphorylation, and hence activation, of tyrosine kinases [3]. The discovery that administration of imatinib mesylate (i.e., Gleevec ${ }^{\circledR}$ ), a TKI, dramatically improved survival in patients with chronic myeloid leukemia (CML) rapidly advanced the development and application of molecular-targeted therapies [4]. Since tyrosine kinases are ubiquitous in distribution, TKIs can adversely affect multiple organs, including the heart [5]. Figure 1 summarizes the main targets of these agents as well as the common mechanisms.

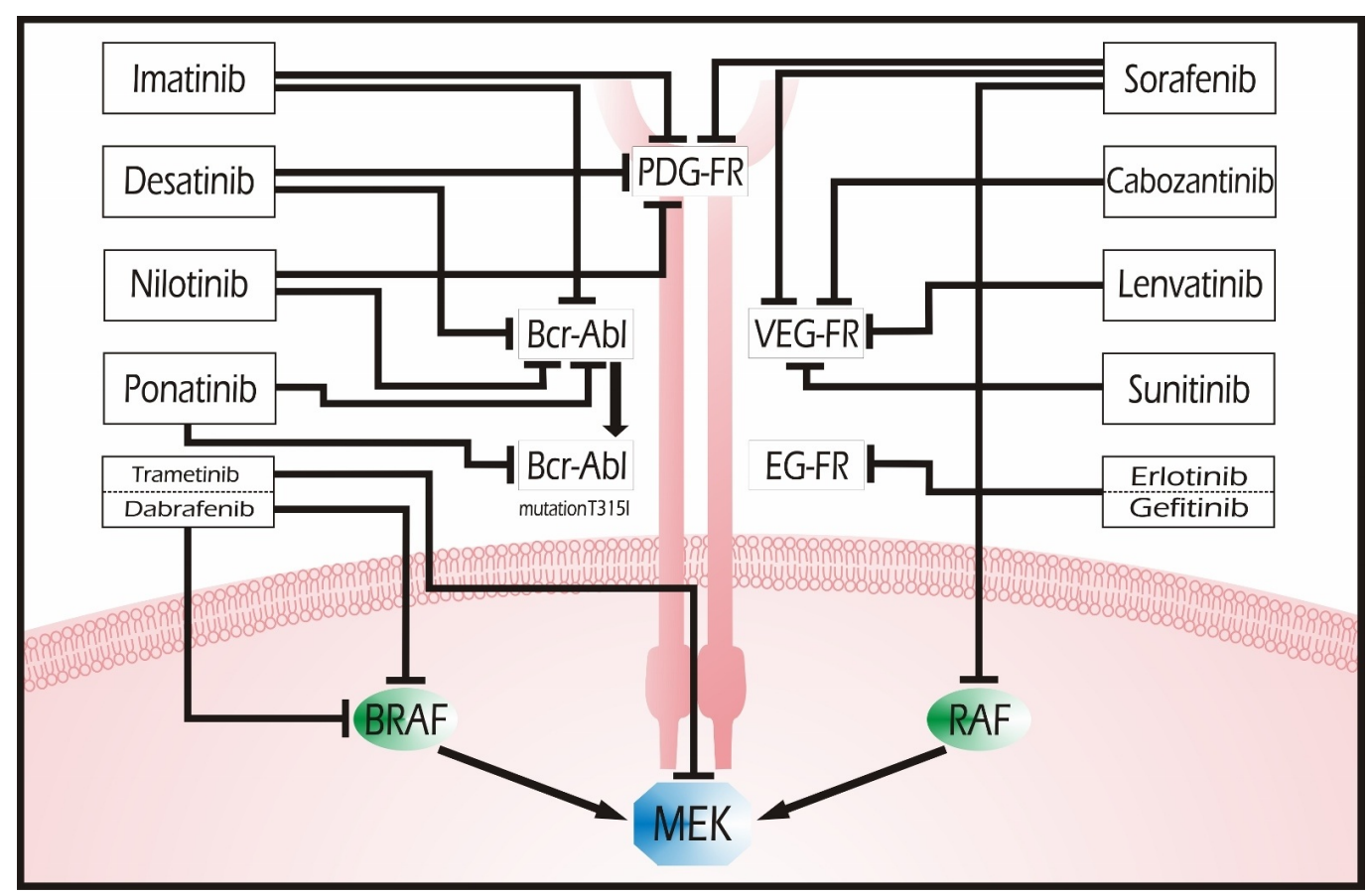

\section{FIGURE 1: Small Molecule Tyrosine Kinase Inhibitors}

The overexpression and/or mutation of tyrosine kinase signaling proteins has been shown to cause abnormal cell proliferation and differentiation, angiogenesis, and inhibition of apoptosis. Tyrosine kinase inhibitors (TKIs) are small molecules that inhibit phosphorylation and, hence, activation of kinases by targeting them at the receptor or intracellular level. Since tyrosine kinases are ubiquitous in distribution, TKIs can adversely affect multiple organs, including the heart. Figure 1 shows the activity of each inhibitor drug on the different kinases. 
Imatinib mesylate targets multiple tyrosine kinases, including Bcr-Abl (the fusion protein encoded by the Philadelphia chromosome), c-Kit (the stem cell factor receptor), and platelet-derived growth factor receptor (PDGFR)- $\alpha$ and $\beta$. It is the drug of choice for the treatment of CML and indicated as the first-line or adjuvant therapy for individuals with Philadelphia chromosome-positive pre-B cell acute lymphoblastic leukemia (B-ALL), gastrointestinal stromal tumors (GIST), and acute and/or chronic eosinophilic leukemia (CEL).

Of patients treated with imatinib monotherapy, $0.5 \%$ to $1.7 \%$ develop heart failure symptoms due to left ventricular (LV) systolic dysfunction [6]. Initial studies that used serum B-type natriuretic peptide or troponin $\mathrm{T}$ levels as a marker of cardiac function reported no cardiotoxicity with imatinib therapy [7]; however, noninvasive imaging studies have demonstrated a decline in the left ventricular ejection fraction with therapy [8]. Although pathological findings characteristic of toxin-induced myopathy have been demonstrated on biopsy in imatinib-treated patients [8], studies have failed to correlate pathologic findings with clinical evidence of cardiac dysfunction. Which patients are most susceptible to developing cardiac dysfunction with imatinib therapy and are candidates for appropriate preventative and management interventions is currently unknown.

\section{Dasatinib}

Currently indicated for the treatment of CML and Philadelphia chromosome (+) ALL (acute lymphoblastic leukemia) after imatinib failure, dasatinib is a very potent TKI targeting Bcr-Abl, cKit, PDGFR- $\alpha$ and $-\beta$, and the Src family of kinases [9]. The most commonly associated adverse cardiovascular effect is peripheral edema. Heart failure incidence is reported to range between $2 \%$ to $4 \%$ [9-10]. Dasatinib treatment is associated with asymptomatic QT interval prolongation ( $\geqslant 500$ $\mathrm{ms}$ ) in $2 \%$ to $3 \%$ of patients, and isolated cases of pericardial effusion have also been reported [6, 10]. Based on similar pharmacodynamic profiles, imatinib and dasatinib-induced cardiotoxicity likely have a common mechanism of action.

\section{Nilotinib}

Nilotinib is an inhibitor of Bcr-Abl, c-Kit, and PDGFR- $\alpha$ and $-\beta$ receptors. Its potency in vitro is 30fold compared to imatinib, and it is used as the second-line treatment in patients with CML initially treated with imatinib and also used in those patients with Philadelphia positive B-ALL. The major cardiac event reported is an increased incidence of QT prolongation (1\% to $10 \%$ incidence), warranting the issuance of a black box warning from FDA as part of the agreement for its approval $[6,11]$. An experiment performed on canine heart myocytes demonstrated that the mechanism behind this action potential delay is an inhibition of phosphoinositide-3-kinase due to "on-target” effects of nilotinib. As a result, multiple ion channels are decreased (including delayed $\mathrm{K}+$ currents IKr and IKs, L-type calcium ion current ICa, L, and peak sodium ion current INa) and persistent $\mathrm{Na}+$ current INaP is increased [12]. Strict monitoring and repletion of magnesium and electrolyte levels are warranted when using this agent.

\section{Lapatinib}

Lapatinib is an orally administered quinazoline targeting epidermal growth factor receptor (EGFR) and ErbB2, which is also the target of the monoclonal antibody, trastuzumab (see below), commonly associated with significant cardiotoxicity. ErbB receptors cause carcinogenesis by complexing with phosphoinositide 3-kinase (PI3K) complexes to activate the serine-threonine kinase Akt pathway [13-14].

In a large randomized trial of combination chemotherapy with lapatinib for metastatic breast cancer, $2.5 \%$ of patients had a marked decrease (> 20\% decline from baseline) in left ventricle ejection fraction (LVEF) without heart failure symptoms [15]. In a pooled analysis of 3,689 patients enrolled in Phase I to III lapatinib clinical trials, $1.6 \%$ of patients experienced a significant decline in LV systolic dysfunction, with $0.2 \%$ being symptomatic [16]. For patients who were previously 
treated with anthracyclines, trastuzumab, or neither prior to lapatinib therapy, the incidence of cardiac events was $2.2 \%, 1.7 \%$, and $1.5 \%$, respectively [16]. The mean time to the onset of cardiac events was 13 weeks, and $88 \%$ of patients followed had a partial or full recovery of LV function regardless of continuation or discontinuation of lapatinib [16]. QT interval prolongation (QTc $>480$ ms or an increase in QTc $>60 \mathrm{~ms}$ from baseline) was not found to be significant in a retrospective study, although the group analyzed only included 16 patients, and one patient increased in common terminology criteria for adverse events [17]. Nevertheless, other studies have found it to occur in up to $16 \%$ of patients [6]. Experiments using a whole-cell patch clamp suggest that this arises from the human Ether-à-go-go-related gene (hERG) channel current delay, which slows heart repolarization. This seems to arise from the inhibition of the coding of the main $\mathrm{IK}_{\mathrm{r}}$ current poreforming subunit [18].

\section{Erlotinib and Gefitinib}

EGFR is overexpressed and/or mutated in many solid tumors [3]. Erlotinib and gefitinib are orally active TKIs targeting EGFR and are used in the treatment of refractory, locally advanced, or metastatic non-small-cell lung cancer after platinum-based chemotherapy [19-20]. Non-smokers, females, Asian descent, and adenocarcinoma phenotype (bronchioalveolar type, in particular) are preferred candidates for these agents [21-23]. Erlotinib is also approved for the treatment of pancreatic cancer in combination with chemotherapy.

In a study of pancreatic cancer patients administered gemcitabine with or without erlotinib, myocardial ischemia or infarction occurred in $2.3 \%$ of patients receiving both and $1.2 \%$ of those who received gemcitabine alone [19]. An increased incidence of deep venous thrombosis (DVT) with erlotinib and gemcitabine combination therapy was also noted (3.9\% vs $1.2 \%$ with gemcitabine, respectively). The mechanism by which these agents contribute to venous thromboembolic events (VTE) remains to be elucidated, but a meta-analysis suggests that the possible mechanism that associates anti-EGFR drugs and thrombosis events is linked to the anti-angiogenic effect of this inhibition. This blockage results in a decrease in production of angiogenic factors, such as VEGF, which enhances the production of nitric oxide (NO). This, in turn, has antiplatelet action and inhibition of leukocyte adhesion that has the potential to expose prothrombotic phospholipids and lead to thrombosis [24].

Additionally, it is important to consider that both erlotinib and gefitinib are agents that are used after platinum-based chemotherapy. A meta-analysis determined that of 932 patients in cisplatin chemotherapy analyzed, $18.1 \%$ developed a thromboembolic event. Of these, $49.7 \%$ exhibited deep vein thrombosis [25]. Thus, it cannot be discarded that there is a possibility that some of the thromboembolic risk attributed to the mentioned TKIs can arise from a predisposition generated by the previous treatment with platinum-based chemotherapy. It has been demonstrated in-vitro that cisplatin has the potential to increase platelet count and endothelial cell damage, which can increase thrombotic events, especially when coupled with gemcitabine treatment [26].

\section{Sunitinib}

Sunitinib is an orally active, multitargeted, antiangiogenic small molecule TKI that inhibits vascular endothelial growth factor receptor (VEGFR), c-Kit, PDGFR- $\alpha$ and $-\beta$, rearranged during transfection (RET) receptor, and FMS-like tyrosine kinase 3 (FLT-3) receptor [27]. It is considered the standard-of-care for treatment of renal cell carcinoma and as a second-line treatment for patients with GIST refractory to imatinib monotherapy [28].

Sunitinib-induced cardiotoxicity is well recognized and the cause of considerable cardiac morbidity (i.e., hypertension, heart failure, myocardial ischemia, etc.) that may not be manifest for weeks or months after completion of sunitinib therapy [29]; the mean time to development of heart failure is highly variable, ranging from 22 days to 27 weeks post-therapy [30-31]. 
Sunitinib inhibits a large number of "direct targets" and "off-targets", which makes it difficult to determine the specific pathway(s) leading to cardiotoxicity. The cardiotoxic effects are mediated, at least in part, through inhibition of PDGFR- $\beta$; microscopic findings compatible with toxin-induced myopathy and mitochondrial damage are present in endomyocardial biopsies from patients with sunitinib-induced heart failure [30]. Cardiomyocyte PDGFR- $\beta$ expression and activity increase in response to pressure overload and regulate myocardial angiogenesis, with PDGFR- $\beta$ knock-out animal models exhibiting impaired stress-induced angiogenesis, myocardial contractile dysfunction, and heart failure [32]. "Off-target” inhibition of AMP-activated protein kinase, ribosomal S6 kinase, and a tyrosine kinase receptor by sunitinib leads to maladaptation to pressure overload (i.e., systemic hypertension), myocyte adenosine triphosphate (ATP) depletion and apoptosis [3, 31]. Maladaptation to pressure loading may be particularly important as hypertension is a common side effect of sunitinib and other VEGFR inhibitors [31]. The mechanism whereby VEGFR antagonists cause hypertension and heart failure is decreased capillary permeability leading to increased cardiac afterload (see also bevacizumab) [33].

LV systolic dysfunction and heart failure are reported in an interval from $3 \%$ to $18 \%$ [34] and symptomatic heart failure in $3 \%$ to $15 \%$ [30-31, 35] of sunitinib-treated patients, with the variability due to the heterogeneous patient populations. Most of these patients with sunitinibinduced cardiotoxicity have coronary artery disease as their only risk factor for heart disease. In clinical studies, heart failure symptoms occurred 22 to 435 days after initiation of sunitinib therapy (average 30.5 weeks) and usually responded well to discontinuation or dose modification of sunitinib and initiation of routine heart failure medical therapy. However, in some individuals, myocardial dysfunction was not reversible despite appropriate therapy.

Other cardiovascular complications associated with sunitinib therapy include elevated serum troponin levels (18\%), MI (2.4\%), and transient ischemic attack (1\%) [30-31]. Approximately onefourth of sunitinib-treated patients develop systemic hypertension (> 150/100 mmHg) with severe (Grade 3 or higher) hypertension occurring in 2\% to $12 \%$ [30, 33, 36]. If hypertension develops, it usually does so with the first cycle of sunitinib and persists throughout treatment [30].

Sorafenib

Sorafenib is a multitargeted, small molecule TKI that inhibits pathways important in cellular proliferation (i.e., RAF-1, B-type Raf [BRAF], and c-Kit) and pathways that are pivotal in tumor angiogenesis (i.e., VEGFR-2, VEGFR-3, FLT-3, and PDGFR- $\beta$ ) [37]. It is currently indicated as a second line for the treatment of renal cell and hepatocellular carcinoma, and as such, it is frequently administered after sunitinib therapy, which raised concerns of cardiotoxicity potentiation [38]. However, a retrospective analysis of 68 patients treated with sorafenib following sunitinib treatment did not reveal increased cardiotoxicity rates with sequential administration [39].

The incidence of acute coronary syndromes, including MI, in patients treated with sorafenib, has been reported to occur in $2 \%$ to $3 \%$ of such individuals [38-42]. In an observational study of 74 patients with metastatic renal cell carcinoma who received either sunitinib, sorafenib, or both by sequential administration, 34\% experienced a cardiac event -- defined as the occurrence of increased cardiac enzymes, symptomatic arrhythmia requiring treatment, new LV systolic dysfunction, or acute coronary syndrome, $40 \%$ had electrocardiographic changes, and $18 \%$ were symptomatic, with $9 \%$ of patients seriously compromised and requiring intermediate care and/or intensive care admission [43].

The pathophysiology of sorafenib-associated cardiotoxicity may be explained by VEGFR and PDGFR inhibition [33, 44-46]. Additionally, RAF inhibition may play a role in its toxicity profile. RAF1 is a member of the RAF family of intracellular signal transducing kinases that inhibit proapoptotic kinases -- MST2 and apoptosis signal-regulating kinase 1 (ASK1) - that regulate oxidant stressinduced injury [47-49]. RAF1 gene deletion in cardiomyocytes results in the development of a 
dilated, hypocontractile heart in animal models [49].

A meta-analysis of 4,599 sorafenib-treated patients reported an overall incidence of hypertension of $23 \%$ with the frequency of Grade 3 or 4 hypertension ranging from $2 \%$ to $31 \%$ [50]. When studied prospectively, a persistent increase in blood pressure was observed within three weeks of sorafenib treatment in most patients, and vascular stiffness increased significantly for up to 10 months of observation [51]. VTE has been associated with sorafenib administration, but the incidence is low $(<2 \%)[33,52]$.

\section{Cabozantinib}

Cabozantinib is a tyrosine kinase inhibitor that is Food and Drug Agency (FDA) approved for the treatment of renal cell carcinoma that has been previously treated with anti-angiogenic therapy. It has inhibitory action over VEGFR2 and tyrosine protein kinase Met (c-MET) [53].

A meta-analysis that revised the development of hypertension in prospective trials with cancer patients following cabozantinib treatment found an incidence of $27.8 \%$ (95\% confidence interval (CI): 23.2 - 32.8\%) for all grade hypertension and 12.0\% (95\% CI: 10.2 - 14.1\%) for high-grade. It is suggested that this occurs through the interaction with VEGFR 2 and VEGF-induced vasodilatory and hypotensive effects. Contrariwise, inhibition of this pathway can produce a hypertensive response [53].

An FDA risk assessment report for cabozantinib indicated that, during the clinical testing program for this drug, no patients suffered torsades des pointes nor were the QTcF > 500 reported [54]. Another FDA risk assessment report wrote that arterial and venous thromboembolism occurred with the administration of cabozantinib in $2 \%$ and $6 \%$ incidence, respectively [55].

\section{Dabrafenib and Trametinib}

Dabrafenib and trametinib are serine-threonine kinase inhibitors that work by blocking the BRAF (dabrafenib and trametinib) and MEK (trametinib only) kinases, which then signal the ERK pathway [56]. Their combined use is FDA approved for the treatment of metastatic melanoma previously diagnosed by an FDA-approved test that detects a mutation in BRAF V600E or V600K. They have a synergistic effect arising from the targeting of different stages of the pathway [57].

Nevertheless, these inhibitors show a series of cardiotoxic complications with their use. For instance, a review that reports on the safety of TKIs establishes that, across clinical trials, evidence for cardiomyopathy (left ventricle dysfunction as measured by the decrease of LVEF > 10\% below baseline) was $11 \%$ when trametinib was administered as a single agent $(n=329)$ and $8 \%$ when combined with dabrafenib ( $\mathrm{n}=202$ ). It also mentions that a study found that the incidence was $9 \%$ $(5 / 55)$ with the combination therapy, while dabrafenib alone showed an incidence of $0 \%$. This suggests that the adverse cardiotoxic effects of dabrafenib might appear only in combination therapy. Correspondingly, this exhibits an average onset of 63 days (range: 16 - 156 days) for single trametinib administration and 86 days (range: 27 - 253 days) for combined treatment [58].

Comparably, the incidence of hypertension (all grades) was found to be $4 \%$ in a clinical trial performed on the adverse effects of combined trametinib and dabrafenib therapy. Crossing over from a trametinib only treatment to a combined one in the same trial led to an incidence of $9 \%$ for all grades of hypertension and 7\% for Grades 3 or 4 [59].

It is suggested that the cardiotoxic effects of these drugs and other BRAF inhibitors arise from blocking of the activation via growth factor of the ERK pathway in cardiomyocytes. This was investigated in perfused rat hearts [56]. 
Lenvatinib is recognized as a multi-targeted tyrosine kinase inhibitor. Its primary activity is over vascular endothelial growth factor receptor (VEGFR), which has an anti-angiogenic effect. This effect accounts for it being FDA approved as a second-in-line treatment for metastatic and progressive thyroid cancer that is refractory to radioiodine (iodine-131), after receiving previous anti-angiogenic therapy. Moreover, it also inhibits other molecular pathways of tumor growth, such as platelet-derived growth factor receptor (PDGFR) and the fibroblast growth factor receptor (FGFR) [60].

One of the major cardiac incidents reported in the use of lenvatinib is the high incidence of hypertension in clinical trials. For instance, a study of this type recognized the appearance of this condition in $69.3 \%(n=261)$ of the subjects for all grade hypertension and $42.9 \%$ for Grades 3 and above [60]. This consequence might arise from a similar mechanism than in cabozantinib administration, where the VEGFR is also inhibited. Less common, although severe, effects reported for the use of lenvatinib in clinical trials were cardiac dysfunction, with a reported incidence of $2 \%$ against $0 \%$ in placebo, and arterial thrombosis, reporting an incidence of $5 \%$ against $2 \%$ placebo (lenvatinib $\mathrm{n}=261$; placebo $\mathrm{n}=131$ ) [61].

\section{Ponatinib}

Ponatinib is indicated in chronic myelogenous leukemia (CML), which is intolerant or resistant to previous treatments. Ponatinib is also used for the treatment of Philadelphia-positive acute lymphoblastic leukemia (ALL) that has the T315I mutation and are resistant to prior therapy with TKIs, such as dasatinib or nilotinib [62-63]. This drug is a third generation inhibitor of the BCR-ABL receptor; its potency relies on the fact that it has clinical activity on both the wild-type and mutated (for example, in CML) BCR-ABL, including the T315I mutation [64].

The two predominant clinical manifestations reported in trials for the use of ponatinib were hypertension and arterial thrombotic events (ATEs). In the case of the first condition, a clinical trial - which involved the analysis of patients with chronic-phase $(n=270)$, accelerated-phase $(n=$ $85)$, and blast-phase $(\mathrm{n}=62)$ CML, and Ph-Positive ALL $(\mathrm{n}=32)$ - determined an incidence of $9 \%$, $7 \%, 2 \%$, and $3 \%$ of all-grade hypertension for each type of cancer, respectively, and $2 \%, 4 \%, 2 \%$, and $3 \%$, also respectively, for Grade 3 or 4 hypertension [65]. In a similar manner, a trial that evaluated the efficiency and safety of ponatinib in 449 CML or ALL (Ph-positive) patients describes that ATEs were present in 19\% of patients, including 10\% cardiovascular, $7 \%$ cerebrovascular, and $7 \%$ peripheral vascular events. Additionally, it reports that venous thromboembolic events were observed in $5 \%$ of the patients. It might be important to mention that this trial had to be temporarily interrupted due to the appearance of ATEs, requiring ponatinib dosage modification before continuing [66].

Furthermore, ponatinib is a TKI that had to be provisionally removed from the market until modifications in the labeling for the safety of thromboembolic events and arterial occlusion were included before its reintroduction [58].

\section{Epigenetic modulators}

Histone deacetylase (HDAC) and acetyltransferases are "epigenetic agents" that regulate the acetylation of histone proteins, thereby activating chromatin transcription at specific gene loci. They also regulate the acetylation of non-histone proteins, including transcription factors involved in cell cycle progression and apoptosis [67-69]. HDAC inhibitors can favorably affect transcription patterns in cancers that exhibit aberrant acetylation patterns that result in (a) transcriptional silencing of tumor suppressor genes [70-71]; (b) inactivation of heat shock protein (HSP) 90 chaperone function; and/or (c) abnormal nuclear factor kappa B (NF-KB) signaling [72] as shown in (Figure 2). 


\section{Cureus}

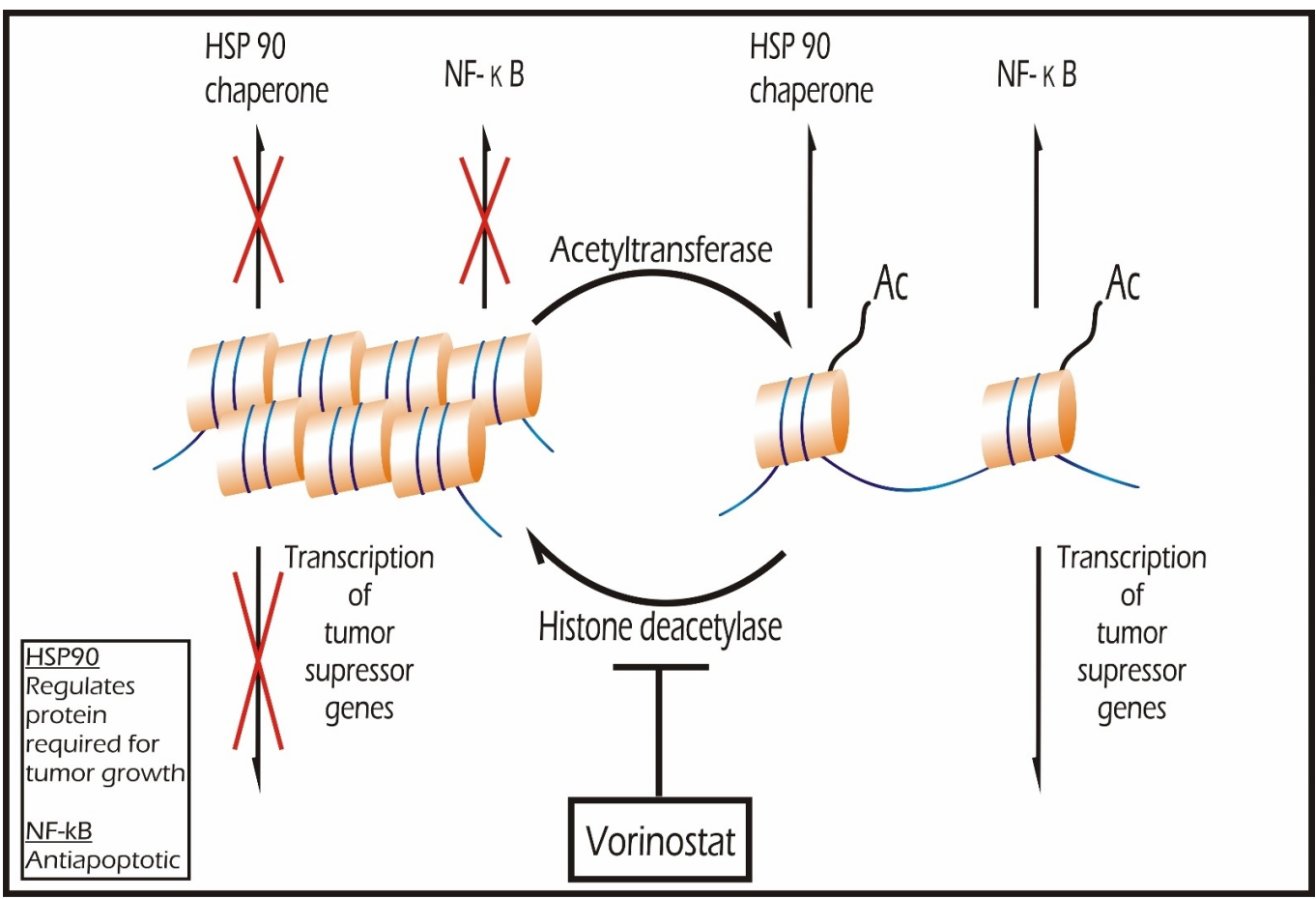

\section{FIGURE 2: Epigenetic Modulators}

Histone deacetylase (HDAC) and acetyltransferases are "epigenetic agents" that regulate the acetylation of histone proteins, thereby activating chromatin transcription at specific gene loci. They also regulate the acetylation of non-histone proteins, including transcription factors involved in cell cycle progression and apoptosis. HDAC inhibitors can favorably affect transcription patterns in cancers that exhibit aberrant acetylation patterns that result in (a) transcriptional silencing of tumor suppressor genes; (b) inactivation of heat shock protein (HSP) 90 chaperone function; and/or (c) abnormal nuclear factor kappa B (NF-kB) signaling.

\section{Vorinostat}

Vorinostat (suberoylanilide hydroxamic acid, SAHA) is an HDAC inhibitor that is currently FDA approved for the treatment of recurrent cutaneous T-cell lymphoma (CTCL) and is under investigation for other (i.e., hematologic) malignancies. In the cardiomyocyte, HDAC influences cardiac hypertrophy, and epigenetic modifications may contribute to cardiac dysfunction and heart failure [73-75]. In dilated cardiomyopathy-derived myocardial human tissue, epigenetic changes in several signaling regulatory pathways have been demonstrated [76].

In patients without known heart disease, vorinostat therapy has been associated with dyspnea in $32 \%$ to $47 \%$ of patients, QT interval prolongation in 3.5\% to 6\%, and thromboembolic events (DVT or pulmonary embolism) in 5\% to $8 \%$ [77-81]. Results of ongoing studies of vorinostat and other cancer therapies targeting epigenetic modifiers are needed to provide further information regarding their cardiotoxicity profile.

\section{Proteasome inhibitors}

Bortezomib

Bortezomib (PS-341) is the first proteasome inhibitor approved by the FDA for the treatment of malignancies. It exhibits antiproliferative and proapoptotic effects on plasma cells and is approved for initial treatment of patients with multiple myeloma [82-83]. As shown in Figure 3, the main target of bortezomib is the ubiquitin-proteasome system (UPS), which is a lysosome-independent 


\section{Cureus}

cellular protein degradation system involved in the regulation of protein expression signaling and proliferation of malignant cell lines [84-86]. Bortezomib also inhibits proteasomal degradation of IKB-alpha, leading to the suppression of the antiapoptotic and proinflammatory transcription factor, NF-KB, and subsequent enhancement of chemotherapy sensitivity [73]. Figure 3 represents the mechanism of action of this drug and its role in the enhancement of chemotherapy sensitivity.

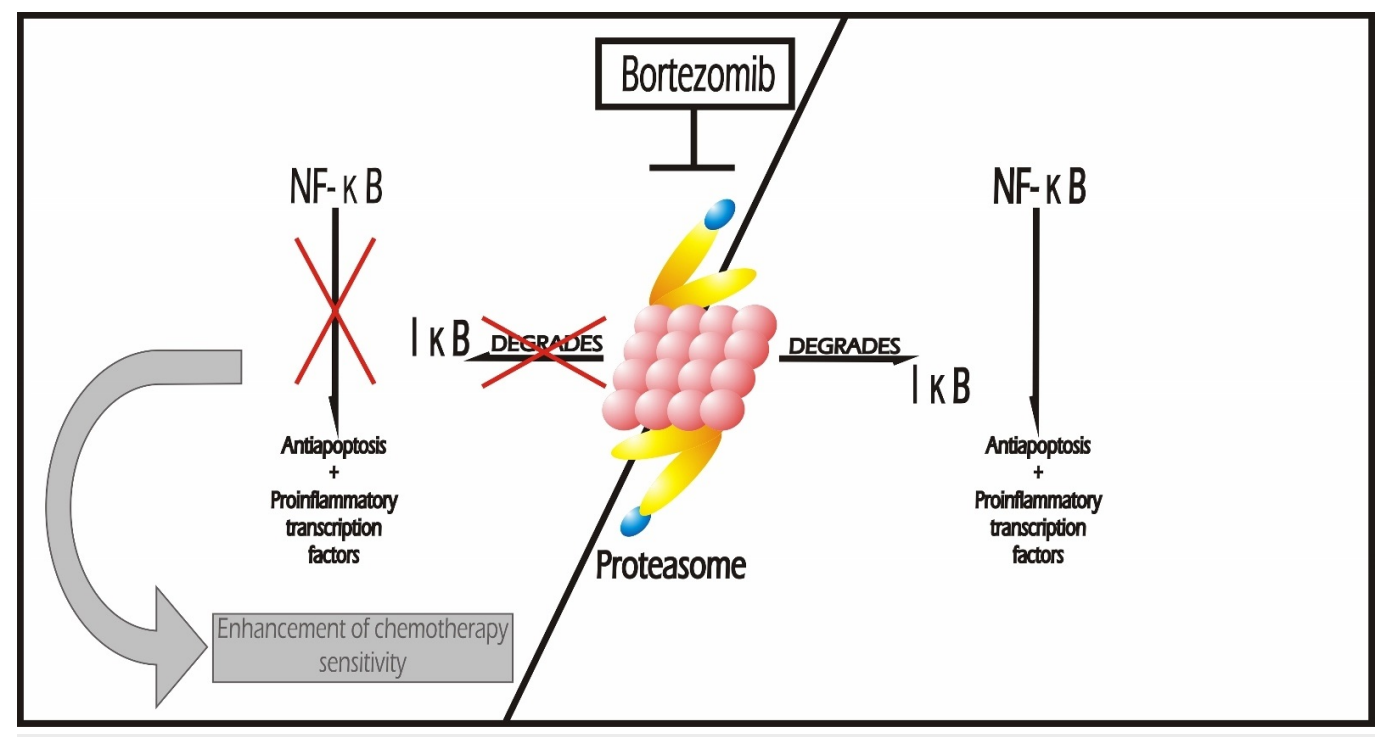

\section{FIGURE 3: Proteasome Inhibitors}

The main target of bortezomib is the ubiquitin-proteasome system (UPS). Bortezomib inhibits proteasomal degradation of IkB-alpha, leading to suppression of the antiapoptotic and proinflammatory transcription factor NF-kN and subsequent enhancement of chemotherapy sensitivity.

Although case reports of bortezomib-induced cardiotoxicity have been published [87-89], the frequency has not been determined. In a Phase III clinical trial of 669 multiple myeloma patients comparing bortezomib with high-dose dexamethasone, the incidence of cardiovascular complications was $15 \%$ versus $13 \%$, respectively, with $2 \%$ of patients in both treatment groups experiencing heart failure [90]. Other proteasome inhibitors (i.e., NPI-0052, CEP-18.770, and RP171) are currently in early phase trials may help to elucidate the clinical impact of UPS disruption on the cardiovascular system $[73,91]$.

Patients suffering from subclinical heart disease are thought to be at particularly increased risk for developing cardiotoxicity with bortezomib treatment [73, 88]. In animal models, UPS is critical for the maintenance of normal cardiac physiology [85, 88], with its impairment leading to the accumulation of oxidized ubiquitinated proteins, which promote cardiomyocyte death and myocardial dysfunction [92]. In ischemia/reperfusion injury models, UPS may be activated as an adaptive mechanism to preserve myocyte and cardiac function [93].

\section{Monoclonal antibodies}

Bevacizumab

Bevacizumab is a chimeric, monoclonal antibody [94] that binds to biologically active isoforms of vascular endothelial growth factor A (VEGF-A), thereby preventing interaction with its endothelial cell receptors (Fms-related tyrosine kinase 1 (FLT-1) and kinase insert domain receptor (KDR)). Bevacizumab is FDA approved for the treatment of various malignancies: it is used in combination with intravenous 5-fluorouracil-based (5-FU) chemotherapy for(a) for first- or second-line treatment of patients with metastatic carcinoma of the colon or rectum; (b) in combination with 
carboplatin and paclitaxel for the first-line treatment of patients with unresectable, locally advanced, recurrent or metastatic non-squamous non-small cell lung cancer; and (c) for the treatment of metastatic renal cell carcinoma in combination with interferon alfa. It is also approved, in combination with paclitaxel, for the initial treatment of patients with metastatic human epidermal growth factor receptor 2 (HER2) negative breast cancer. Lastly, it has recently been approved for treatment of glioblastoma, as a single agent for patients with progressive disease following prior therapy [95].

As with other anti-VEGF targeted therapies, hypertension is a common adverse effect in patients treated with bevacizumab monotherapy or in combination with other targeted agents. In clinical trials, the incidence of bevacizumab-induced hypertension has been reported between $4 \%$ to $35 \%$ [6, 33, 96-103], with Grade 3 hypertension reported in $11 \%$ to $18 \%$ of patients $[6,96-98,100,104-$ 105] and approximately $2 \%$ of patients having severe (Grade 4 ) hypertension requiring discontinuation of the drug [106]. The median interval from the initiation of bevacizumab therapy to the development of hypertension (HTN) is 4.5 to 6 months [33]. Interestingly, the development of hypertension during bevacizumab therapy is considered a favorable prognosticator, as it denotes the presence of certain "hypertension-susceptible” VEGF polymorphisms (VEGF-2578 AA and VEGF-1154 A) linked to a better response to chemotherapy and increased survival [107]. Higher doses and concomitant therapy with sorafenib are associated with an increased incidence of hypertension [33, 108-109].

Cardiac dysfunction and heart failure are potential adverse effects of therapy with bevacizumab, with a reported incidence of heart failure ranging from 1.7\% to 3\% [95-97]. Patients previously exposed to traditional therapies known to cause cardiomyopathy, such as anthracyclines [96, 110], mitoxantrone [111], or capecitabine [96], are at particular risk of suffering this complication through potentiation of their cardiotoxic effects.

The mechanism of bevacizumab therapy-related HTN is thought to be related to microvascular rarefaction and inhibition of the nitric oxide-mediated vasomotor effects of VEGF. Microvascular rarefaction is an anti-angiogenic effect leading to the extinction of the small arterioles and capillaries that comprise the microcirculation, and it is a common finding present in individuals suffering from arterial hypertension [112]. The presence of this phenomenon associated with bevacizumab therapy was documented in a prospective study demonstrating a decline in the mean dermal capillary density after six months of treatment and its association with the development of hypertension [113].

Inhibition of VEGFR-2 activation with bevacizumab leads to blockage of endothelial nitric oxide synthase up-regulation through the Src and Akt signaling pathways. The resultant decreased nitric oxide production promotes vasoconstriction and increases peripheral vascular resistance [114].

Bevacizumab-induced heart failure is closely related to uncontrolled hypertension and the cardiac remodeling response. VEGF signaling in cardiomyocytes is a major mediator, not only in angiogenesis but also in compensatory responses to pressure load and injury [33, 115-116]. In animal models mimicking bevacizumab anti-VGEF effects, pressure overload results in the reduction of myocardial capillary density, global contractile dysfunction, cardiac fibrosis, and eventually decompensated heart failure [117].

Venous and arterial thromboembolic events, including angina pectoris, myocardial ischemia, or infarction and cerebral infarction, occur at a higher incidence in patients treated with bevacizumab, plus chemotherapy, as compared with those treated with chemotherapy alone [95]. A meta-analysis of 15 randomized trials demonstrated a 33\% increased risk of developing VTE associated with bevacizumab treatment (relative risk: 1.33; $\mathrm{p}$.001) [118]. A pooled analysis of five randomized clinical trials demonstrated an incidence of arterial thrombotic events of $3.8 \%$ in the bevacizumabtreated patients and 1.5\% developed myocardial infarction or ischemia [119]. An observational study reported serious arterial thrombotic events in $1.8 \%$ and $\mathrm{MI}$ in $0.6 \%$ of bevacizumab-treated patients [120]. These events tend to occur at any time during therapy, with a median time-to-event 


\section{Cureus}

of about three months and are not dose-dependent. A history of prior vascular thrombosis and age $>65$ years have been identified as potential risk factors [119-120].

VEGF plays a significant role in the maintenance of vascular integrity through the stimulation of endothelial cell proliferation and preservation of endothelial cell junctions [121]. VEGF inhibition with bevacizumab promotes endothelial cell dysfunction and apoptosis and decreases the endothelial regenerative potential, which predisposes to both hemorrhagic and thrombotic events, especially in the setting of trauma [114, 121]. Platelet activation and aggregation due to subendothelial collagen exposure and subsequent tissue factor activation are key factors in the prothrombotic cascade [114]. Additionally, reduction of nitric oxide and prostacyclin promote vasoconstriction and thrombosis [33, 114].

\section{Trastuzumab}

Trastuzumab is a chimeric, monoclonal IgG antibody against the extracellular domain of HER2 [122-123]. HER2 protein overexpression is observed in $20 \%$ to $35 \%$ of primary breast cancers [122-125] and is associated with poorer outcomes [124]. Trastuzumab, as a single agent or in combination with immunochemotherapy, improves outcome in breast and gastroesophageal cancer patients who overexpress HER2 [124, 126]. The risk of recurrence and mortality are reduced when trastuzumab is integrated into adjuvant chemotherapy for early stage localized breast cancer that overexpresses HER2 [124, 127]. As a result, many breast cancer patients who are treated with trastuzumab receive anthracyclines before or simultaneously.

LV systolic dysfunction is the most common cardiotoxic effect induced by trastuzumab, with the mechanism, pathologic findings, and clinical outcome different than anthracycline-induced cardiac dysfunction. The cardiotoxic effects of the trastuzumab are not cumulative or dose-related, as seen with anthracyclines. Although the risk of cardiomyopathy is increased in patients who have been treated with both agents, some develop heart failure during treatment with trastuzumab in the absence of exposure to anthracyclines [125, 127-129]. Endomyocardial biopsies have revealed two types of chemotherapy-induced cardiac dysfunction (Table 1) [128-129]. Type I cardiotoxicity is characteristic of anthracycline exposure with myocyte damage on pathologic biopsy, clinical heart failure, and minimal or no improvement in ventricular function with cessation of therapy. Type 2 cardiotoxicity is characterized by reduced contractility with little myocyte necrosis on microscopic examination and frequent improvement in ventricular function with cessation of therapy [128].

Type I CRCD (model: doxorubicin)

Cellular death

Myocyte necrosis and typical ultrastructural changes on light and electron microscopy

Cumulative dose-related

Permanent damage

\section{Type II CRCD (model: trastuzumab)}

\section{Cellular dysfunction}

No injury or myonecrosis by light and electron microscopy

Not cumulative dose-related

Generally reversible with cessation of drug

\section{TABLE 1: Clinical Features Distinguishing Type I and Type II Chemotherapy- related Cardiac Dysfunction (CRCD)}

$[128,130]$ 
electron microscopic evidence of injury [5, 128], suggesting that trastuzumab depletes adenosine triphosphate by impairing mitochondrial function without permanently altering myofibrillar ultrastructure [131]. Alternatively, the cardiotoxicity associated with HER2 receptor blockade may result from a decreased ability to mount an integral response to stress [127-128]. Signal transduction via epidermal growth factors is fundamental in regulating the hypertrophic response to myocytes and the sarcomeric organizational response towards different stimuli, including protection against cardiac toxins [127-128, 132]. The HER2 gene knock-out mice have a higher sensitivity for anthracycline-associated cardiotoxicity and the development of progressive heart failure and premature death compared to wild-type mice [127-128, 133].

It is postulated that trastuzumab induces cardiotoxicity in hearts susceptible to dysfunction as a result of prior or concomitant anthracycline treatment (the so-called "two-hit theory") [132] by interfering with the repair of myocytes damaged by anthracycline exposure.

In a pooled analysis of 1,219 patients enrolled in Phase II and III clinical trials, LV systolic dysfunction was noted in $9.2 \%$ of those who received trastuzumab, and the incidence was increased when trastuzumab was administered concurrently with anthracyclines. Severe heart failure symptoms (New York Heart Association (NYHA) Class III to IV) were present in 16\% of trastuzumab-treated patients who received an anthracycline concomitantly and only $2 \%$ of those who received paclitaxel concomitantly $[127,134]$. Of the patients who developed symptomatic heart failure with trastuzumab therapy, LV function normalized in $79 \%$ when the agent was discontinued and appropriate heart failure therapy initiated. Cardiotoxicity reversed quickly (average: 1.5 months) [134]; when trastuzumab therapy was reinitiated in 25 patients, only three $(12 \%)$ had a recurrence of LV dysfunction $[5,135]$.

A pooled analysis of randomized controlled trials and case-control studies showed that the prevalence of cardiotoxicity in the trastuzumab-treated patients was $10 \%$ whereas, in the nontrastuzumab comparator arm, the prevalence was $2 \%$ [136]. In a meta-analysis of five randomized controlled trials, a $10 \%$ decline in LV ejection fraction was observed in $3 \%$ to $34 \%$ of trastuzumabtreated patients $[128,137]$.

Independent risk factors for trastuzumab-induced cardiotoxicity are simultaneous or prior exposure to anthracycline and increased patient age $[6,125,128,138]$. Similar to anthracycline-induced cardiotoxicity, previous cardiac disease and NYHA Class II symptoms are suspected risk factors [6]. However, traditional cardiac risk factors, prior cardiac disease, prior chest radiation, and preexisting hypertension have not been identified as risk factors for trastuzumab-induced cardiac dysfunction [6, 128]. In patients receiving concurrent anthracycline and trastuzumab therapy, the risk of cardiac dysfunction increases after the cumulative dose of doxorubicin exceeds $300 \mathrm{mg} / \mathrm{m}^{2}$ $[6,127,137-138]$.

\section{Rituximab}

Rituximab is a chimeric murine/human monoclonal antibody that binds the cluster of differentiation 20 (CD20) protein, which is expressed on the surface of B cells [139]. CD20 functions as an ion channel essential for regulating cell cycle progression and calcium homeostasis.

Stimulation of the CD20 receptor induces depletion of intracellular calcium stores, thereby affecting calcium-dependent signaling processes, such as transcriptional control, cell cycle progression, and apoptosis [139-140].

Rituximab is indicated for the treatment of various non-Hodgkin's lymphomas, either alone or in combination with other chemotherapeutic agents [141-142]. The major cardiovascular side effect observed with Rituximab therapy is hypotension, which occurs in up to $10 \%$ of patients [125]. It typically occurs in the first few hours of the drug's initial infusion and is responsive to fluid therapy $[125,141-143]$. The exact mechanism in which the cardiovascular system is affected is unknown, 
but it is likely related to rituximab's calcium channel blocking function. Despite the acute effects, there is no increased risk of cardiotoxicity in patients with non-Hodgkin's lymphoma when rituximab is added to standard (i.e., CHOP - cyclophosphamide, hydroxydaunorubicin, oncovin, and prednisone) chemotherapy [143].

\section{Alemtuzumab}

Alemtuzumab, a humanized IgG1 directed against the CD52 protein, is primarily indicated in patients with chronic lymphocytic leukemia (CLL) or small cell lymphoma [125, 144-145]. It is also used in patients with immune-mediated, nonmalignant conditions, such as rheumatoid arthritis, solid organ transplants, multiple sclerosis, and as a conditioning agent for bone marrow transplantation [144].

Alemtuzumab has been associated with infusion-related reactions, including hypotension, bronchospasm, and rash, typically during the first week of therapy [125, 142]. LV dysfunction is rare but has been reported in patients with cutaneous T-cell lymphoma who had previously received multiple chemotherapy regimens [125, 146]. The mechanism is not fully understood [146]. Close monitoring for hypotension is recommended for patients with the preexisting cardiac disease who are treated with this agent [125].

\section{Ibritumomab Tiuxetan}

Ibritumomab tiuxetan is an agent used in patients with relapsing or refractory low-grade follicular transformed B-cell non-Hodgkin's lymphoma [144]. It is composed of an anti-CD20 mouse antibody (i.e., ibritumomab) chemically attached to a chelator linked to the beta-emitting isotope yttrium90 [147-148].

Hypotension and cardiac arrhythmias are rare complications associated with ibritumomab infusion $[125,142]$. Since ibritumomab tiuxetan is administrated in combination with rituximab[147], it is unknown if the adverse cardiovascular reactions are the result of one or the other agents or their interaction. Additionally, the long-term cardiac effects of local beta-irradiation are unknown.

\section{Tositumomab}

Tositumomab is an IgG2a anti-CD20 monoclonal antibody derived from immortalized mouse cells. It is administrated in a sequential infusion followed by iodine131 (131I) tositumomab (i.e., the antibody linked to I131 by a covalent reaction) which emits both beta and gamma radiation [149150]. It is indicated for the treatment of patients with CD20 antigen-expressed refractory, low-grade, follicular or transformed non-Hodgkin's lymphomas, and in patients with rituximab-refractory nonHodgkin's lymphomas [144, 149].

Hypotension (7\%), peripheral edema (9\%), chest pain (7\%), and vasodilatation (5\%) are cardiovascular complications that have been described with the use of this antineoplastic compound [149]. Due to its radioactive emissions, studies assessing the potential cardiovascular effects of this radio-immunotherapeutic agent are still needed.

\section{Cetuximab}

Cetuximab, a human/mouse chimeric monoclonal IgG1 antibody that binds to human EGFR, is currently used to treat colorectal $[125,144,151]$ and head and neck cancer [151]. Cetuximab blocks phosphorylation and activation of receptor-associated kinases, resulting in the inhibition of growth and survival of tumor cells that overexpress EFGR [152]. 
Potentially fatal infusion reactions involving severe hypotension have been described in approximately $3 \%$ to $5 \%$ of patients receiving this medication $[125,142,144]$.

\section{Panitumumab}

Panitumumab is a recombinant human IgG2 kappa monoclonal antibody to EGFR [153] that has been approved for EGFR-expressing metastatic colorectal carcinoma with disease progression on or following fluoropyrimidine, oxaliplatin, and irinotecan-containing chemotherapy regimens.

Panitumumab and cetuximab have the same target receptor but different IgG isotypes, which may convey different ligand affinities and cardiotoxicity profiles. Peripheral edema is the most common cardiovascular side effect, occurring in $12 \%$ of panitumumab-treated patients [153]. As the use of this agent increases in patients with RAS and BRAF wild-type colorectal cancers, the cardiotoxic effects common to other agents that target the EGFR ligand (e.g., erlotinib, lapatinib, etc.) could also be noted in these patients.

\section{Ofatumumab}

Ofatumumab is an IgG1-kappa monoclonal antibody that binds to the CD20 molecule resulting in B-cell lysis [154]. This agent is FDA approved for the treatment of patients with CLL refractory to fludarabine and alemtuzumab [154]. Its role in the treatment of follicular non-Hodgkin's lymphoma, diffuse B-cell lymphoma, rheumatoid arthritis, and multiple sclerosis is currently under investigation.

Reported adverse cardiovascular reactions include peripheral edema (9\%), hypertension (5\%), hypotension (5\%), and tachycardia (5\%) [154]. The pathophysiology of these side effects may be related to its interaction with $\mathrm{CD} 20$-like ligands in noncancerous tissues.

\section{Lenalidomide}

Lenalidomide is a thalidomide analog possessing immunomodulatory and antiangiogenic properties [149]. Lenalidomide is FDA approved for the treatment of myelodysplastic syndrome associated with chromosome $5 q$ deletion and multiple myeloma, in combination with dexamethasone, in patients who have received at least one prior therapy [155-156]. Its exact mechanism of action is not fully understood, but it inhibits cell proliferation and affects inflammatory cytokines in vitro [156].

The most common cardiovascular adverse reactions associated with this agent are peripheral edema (20\% incidence), atrial fibrillation (2.9\%), and VTE. The latter varies in incidence from $3 \%$ to $75 \%$ [6, 156]. A black box FDA warning is included in the package insert for this medication indicating that patients with multiple myeloma who receiving lenalidomide combination therapy may benefit from simultaneous thromboembolism prophylaxis or aspirin [157]. Administered as a single agent, it is not associated with an increased risk of thrombotic events $[6,158]$.

\section{Checkpoint inhibitors}

Nivolumab and Pembrolizumab

Nivolumab and pembrolizumab are two checkpoint inhibitors that work on PD-1 receptors to trigger T-cell activation. When T-cells reach cancer cells, inactivation of this pathway can happen due to the binding of the ligand PD-L1 to the mentioned receptor [159] as schematized in Figure 4. Therefore, the use of these drugs serves as immunotherapy against tumor cells. These are FDA approved for the treatment of (a) non-small cell lung cancer that has metastasized and (b) melanoma that cannot be removed by surgery. Additionally, nivolumab is approved for the treatment of classical Hodgkin lymphoma and advanced renal cell carcinoma. 


\section{Cureus}

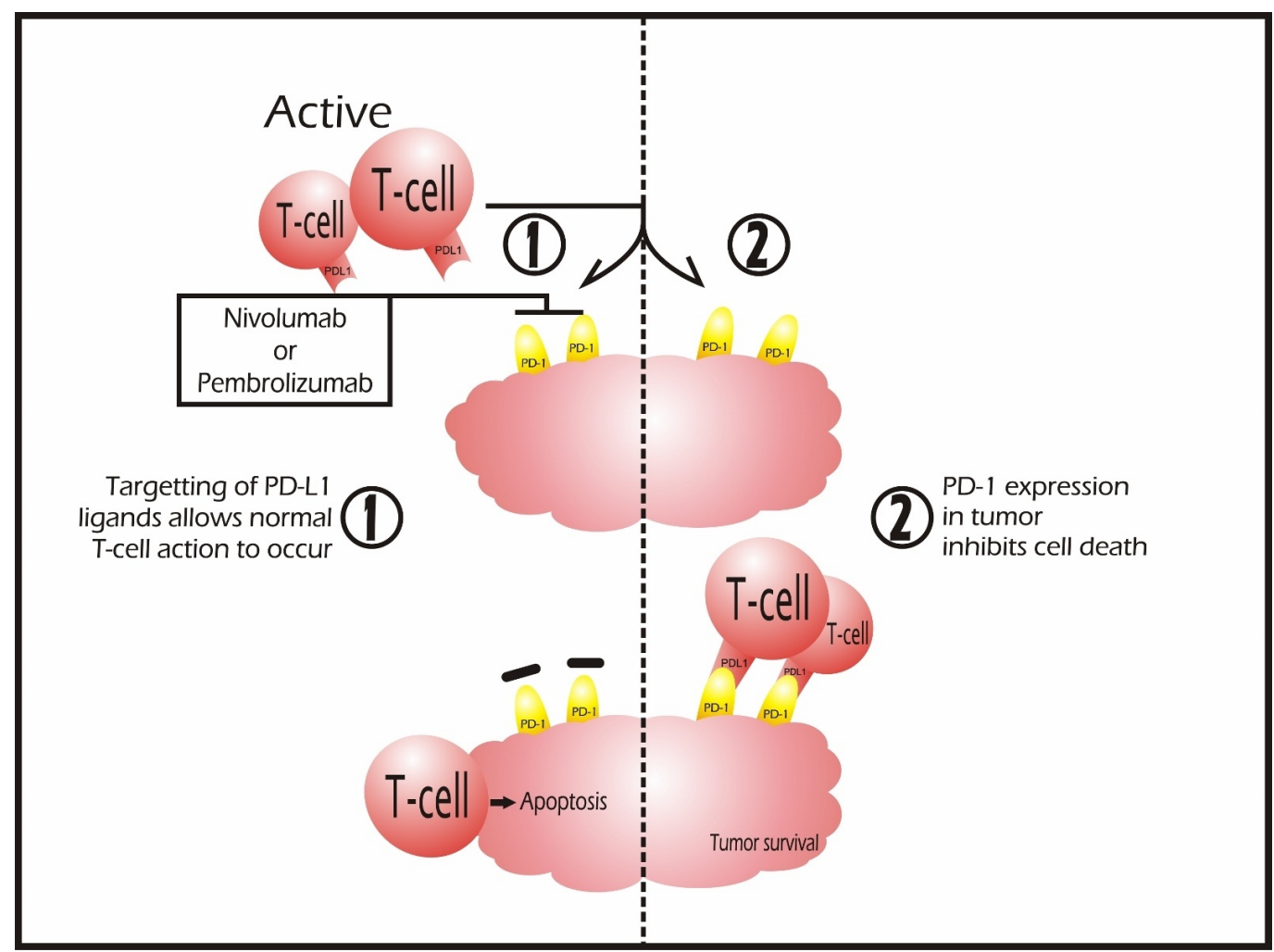

\section{FIGURE 4: Checkpoint Inhibitors}

Nivolumab and pembrolizumab are two checkpoint inhibitors that work on PD-1 receptors to trigger Tcell activation. When T-cells reach cancer cells, inactivation of this pathway can happen due to the binding of the ligand PD-L1 to the mentioned receptor. Therefore, the use of these drugs serves as immunotherapy against tumor cells.

The incidence of cardiotoxicity of checkpoint inhibitors is reported to be very low in early clinical trials. For instance, a review reported that only $2 \%$ of 296 patients who received $10 \mathrm{mg} / \mathrm{kg}$ of nivolumab exhibited hypotension. Similarly, only $7 \%$ of 135 melanoma patients who received 10 $\mathrm{mg} / \mathrm{kg}$ of pembrolizumab showed a development of hypertension [160]. Cardiotoxic mechanisms are still to be elucidated, but mouse models suggest that heart dysfunction and dilation occur as an autoimmune response to cardiac troponin I in PD-1 deficient mice through chronic stimulation of $\mathrm{Ca} 2+$ influx [161]. It might be relevant to assess potential cardiotoxic effects from PD-1 ligand inhibition as treatment with these checkpoint inhibitors is expected to expand to several other cancer types.

\section{mTOR inhibitors}

Figure 5 exhibits simplified pathways for the mammalian target of rapamycin and the roles it serves in cell cycle progression, transcription, and other factors related to proliferation. 


\section{Cureus}

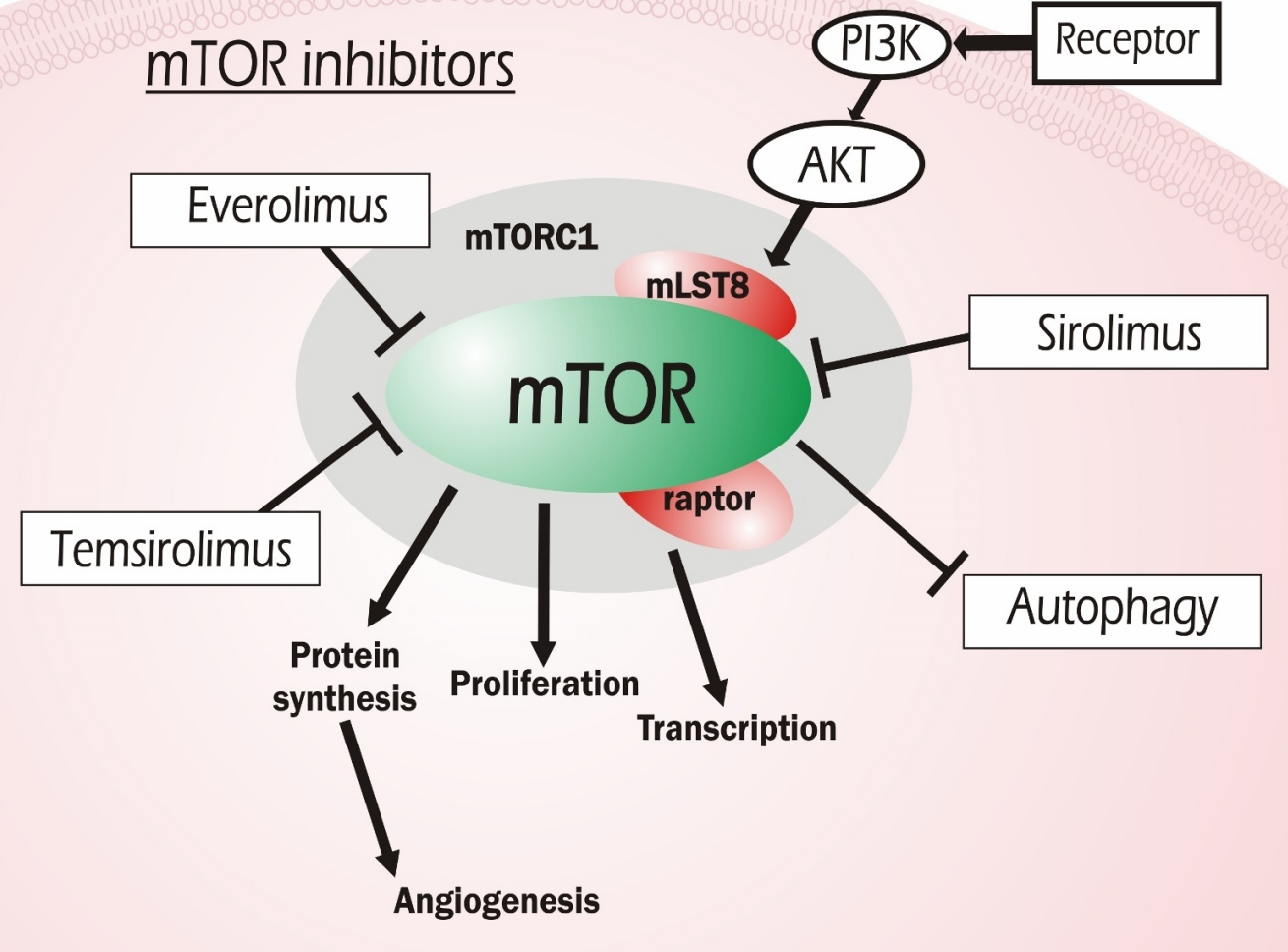

\section{FIGURE 5: mTOR Inhibitors}

The target of these agents is the mammalian target of rapamycin (mTOR), a key regulatory kinase that interferes with cell cycle progression from the $\mathrm{G} 1$ to $\mathrm{S}$ phase via the formation of an immunosuppressive complex with FK binding protein 12 (FKBP-12). Inhibition of this pathway by everolimus, sirolimus, or temsirolimus reduces possible angiogenesis and proliferation of cells.

Sirolimus

Sirolimus inhibits (a) T-lymphocyte activation and proliferation in response to antigenic and cytokine stimulation and (b) antibody production by inhibiting the mammalian target of rapamycin (mTOR), a key regulatory kinase that interferes with cell cycle progression from the G1 to S phase, via the formation of an immunosuppressive complex with FK binding protein-12 (FKBP-12) [162163]. This agent is used to prevent rejection in solid organ transplantation and treat Kaposi sarcoma in renal transplant patients.

Since this medication and its derivatives (discussed below) have a complex mechanism of action -interacting with various growth factors, the redox state of the cell, transcription and protein synthesis, and cell survival -- the cardiovascular effects are also complex and not fully elucidated. In the mice model, mTOR expression preserves cardiac function by controlling collagen generation, attenuating fibrosis [164], and suppressing cytokine-mediated pathways responsible for cardiac dysfunction [165]. In animal models, rapamycin (or everolimus) interferes with post-MI LV remodeling through augmentation of autophagy [166].

Clinical studies have demonstrated dose-related cardiovascular effects in $\geqslant 20 \%$ of patients treated with rapamycin, including peripheral edema (in 54\% to 64\%), hypertension (in $39 \%$ to $49 \%$ ), peripheral edema (in $16 \%$ to $24 \%$ ), and chest pain (in 16\% to $24 \%$ ) of affected individuals. Severe cardiovascular effects -- atrial fibrillation, heart failure, DVT, and hypotension -- are uncommon, with a reported incidence of $<3 \%$ [162]. 
Everolimus, a derivative of sirolimus, is FDA approved for the treatment of patients with advanced renal cell carcinoma (RCC) after failed treatment with sunitinib or sorafenib and as prophylaxis for renal transplant rejection [167]. Through the formation of a complex with the FKBP-12 protein, it inhibits mTOR and subsequent phosphorylation of P70 S6 ribosomal protein kinase, thereby preventing protein synthesis and cell proliferation [167]. Additional antiproliferative effects are exerted by decreasing eukaryotic elongation factor 4E-binding protein and expression of VEGF and hypoxia-inducible factor [167].

In clinical trials, patients treated with everolimus experienced hypertension (4\% of advanced renal cancer patients and $30 \%$ of kidney transplant recipients), peripheral edema (25\% of advanced renal cancer patients and $45 \%$ of kidney transplant recipients), and tachycardia rarely [167].

\section{Temsirolimus}

Temsirolimus, also a derivative of sirolimus, leads to G1 phase cycle cell arrest and exerts antiangiogenic properties by reducing the synthesis of VEGF. It is FDA approved for the treatment of advanced renal cell carcinoma [168]. Cardiovascular side effects associated with its use include hypertension (7\%), and VTE (2\%) [168]. Figure 5 presents the mechanism of action of the mTOR inhibitors herein described.

\section{Prevention and treatment}

Increased awareness of the potential cardiovascular side effects associated with the various chemotherapeutic agents allows early detection and appropriate treatment. Cardiac events associated with newer agents have a highly variable incidence and onset, ranging from days to months after the treatment is administered. Oncologists, cardiologists, and primary care physicians should educate patients about potential cardiotoxicity associated with chemotherapy, risk factors, the need for ongoing monitoring during administration of chemotherapy, and long-term follow-up to assess for late cardiovascular complications. Although the Heart Failure Society guidelines do not recommend reevaluation of cardiac function on a regular basis, monitoring of LV function at regular intervals should be strongly considered since many patients who develop decreased LV ejection fraction are asymptomatic [169]. Regular monitoring of cardiac function is particularly important in individuals considered to be at increased risk of chemotherapy-induced cardiotoxicity or those receiving an agent or agents with a high incidence of cardiotoxicity.

For patients receiving trastuzumab, some oncology centers propose that patients with elevated risk (i.e., elderly, reduced LV ejection fraction, cardiac risk factors present, etc.) undergo a clinical and echocardiographic assessment every three months while receiving chemotherapy and then every six months for five years subsequently [169-170]. For patients without elevated risk, the assessments can be performed every six months until the conclusion of trastuzumab therapy and yearly thereafter for three years. If new symptoms occur or if the LV ejection fraction declines by more than $10 \%$, cessation of treatment - at least transiently -- may be necessary. If late cardiac complications related to trastuzumab are found, longer term monitoring may be appropriate [128, 171].

Newer noninvasive imaging modalities (i.e., speckle tracking echocardiography) may allow earlier detection of chemotherapy-induced cardiac dysfunction, even before frank systolic dysfunction occurs [172]. Efforts to identify biomarkers, such as serum cardiac isoenzymes, including troponin and/or brain natriuretic peptide, for early diagnosis of chemotherapy-induced cardiotoxicity and follow-up of this entity are also under investigation [128, 169, 171, 173].

Once a cardiovascular complication has been detected, efforts should be aimed to minimize the 
progression of cardiac and endothelial dysfunction. This may require pharmacokinetic changes, switching to chemotherapeutic analogs that are less cardiotoxic, administering cardioprotective agents (e.g., dexrazoxane), and avoiding additional cardiotoxic regimens. In many patients, discontinuation of the chemotherapeutic agent is the only available option, which might not be feasible or acceptable to the patient due to the risk of cancer progression.

With regard to pharmacologic therapy, evidence-based management guidelines have not been established, although most experts initiate standard therapies based on presumed benefit. These treatments may include antihypertensive medications, diuretics, renin-angiotensin-aldosterone system (RAAS) blockers, beta-blockers, antiplatelet agents, or antiarrhythmics.

Treatment of hypertension should be aimed to reduce morbidity and mortality and lower the risk of associated end-organ damage. Hypertension induced by VEGF targeting agents is highly responsive to antihypertensive therapy, which means that interruption of chemotherapy is not usually necessary. Angiotensin-converting enzyme (ACE) inhibitors or angiotensin receptor blockers (ARBs) are the initial treatment of choice due to their potential to increase nitric oxide release and to reduce bradykinin catabolism, plasminogen activator inhibitor-1 expression, microcirculatory changes, and proteinuria [102, 174-175]. An additional benefit of RAAS inhibitors may be potentiation of the antiangiogenic effects of VEGF-based therapy since angiotensin II-IV (downstream cleavage products of angiotensinogen) upregulates VEGF in tumor tissue [176].

In some individuals, additional antihypertensive medications may be required to control hypertension [175]. If such is the case, non-dihydropyridine calcium channel blockers (e.g., verapamil and diltiazem) should not be used in combination with cytochrome P450 3A4 (CYP3A4) isoenzyme inhibitors (e.g., sorafenib), due to the risk of markedly increased concentrations of the chemotherapeutic agent. If therapy with a calcium-channel blocker is desired, amlodipine or nifedipine are preferred.

If cardiac systolic dysfunction develops, the offending chemotherapeutic agent(s) should be discontinued until the patient has been stabilized and started on appropriate heart failure-based therapy according to guidelines published by the American College of Cardiology, American Heart Association, and Heart Failure Society of America [173]. An ACE inhibitor or ARB, in combination with a beta-blocker, is recommended unless contraindicated. In patients with anthracycline-induced cardiomyopathy, enalapril therapy can reduce the decline in LVEF and subsequent cardiac events [177]. Valsartan, an ARB, blocked the acute cardiotoxic effects of anthracycline treatment during a small randomized trial [178]. Among the beta-blocking agents, carvedilol demonstrated cardioprotection in anthracycline-treated patients, probably by virtue of its intrinsic antioxidant properties [179]. In addition to heart failure therapy, treatment should be instituted for any comorbid conditions that may adversely affect cardiovascular function (i.e., hypertension, diabetes, and hyperlipidemia). Optimal management of hypertension is pivotal to prevent heart failure progression.

Patients with asymptomatic bradycardia or QT interval prolongation as a result of chemotherapy should continue therapy with ongoing monitoring. Conversely, symptomatic patients may require discontinuation of the offending agent (including beta-blockers, calcium channel blockers, and digoxin) and placement of a permanent pacemaker if advanced heart block is present [79]. Multiple myeloma patients who develop symptomatic bradycardia with thalidomide therapy should be considered for pacemaker implantation [180].

Treatment of myocardial ischemia in the setting of chemotherapy may include medical therapy, such as beta blockers, calcium channel blockers, statins, antiplatelet agents, and anticoagulants, and percutaneous coronary intervention (PCI) [181-182]. If $\mathrm{PCI}$ is performed, the procedure choice - balloon angioplasty, placement of a bare metal stent, or a drug-eluting stent) -- may be determined by whether the patient can receive dual antiplatelet therapy for an extended period 
(i.e., six to 12 months, in which case placement of a drug-eluting stent can be considered) or for only a limited time (up to four weeks), in which case a balloon angioplasty or placement of a baremetal stent is advised.

The decision to administer therapy to prevent VTE events should be individualized based on the patient's risk factors. Aspirin may be used in selected patients considered to be at increased risk for arterial and VTE complications, especially if platelet count and function are preserved [119]. The International Myeloma Working Group recently issued recommendations regarding the prevention of thalidomide- and lenalidomide-associated thrombosis in myeloma patients [183]. Aspirin (81 to $325 \mathrm{mg}$ ) is recommended for low-risk patients, and low-molecular-weight heparin (LMWH), enoxaparin $40 \mathrm{mg}$, or full-dose Warfarin is recommended for those with two or more risk factors or receiving concomitant high-dose dexamethasone or doxorubicin. The risk factors include age, history of VTE, central venous catheter, comorbidities (infections, diabetes, and cardiac disease), immobilization, surgery, inherited thrombophilia, and hyperviscosity.

Once a VTE is diagnosed, the LMWH (enoxaparin, dalteparin, or nadroparin) should be administered for three to six months, followed by anticoagulant therapy with warfarin or LMWH indefinitely or until cancer remission is achieved [184].

\section{Radiotherapy}

It must be highlighted that radiotherapy itself may be cardiotoxic, so conjunction with chemotherapy that has the same effects may worsen the risk. Nevertheless, further studies need to be done with regard to the combined use of each drug and radiotherapy. This is especially salient in agents like tositumomab, which combine radiation and targeting by monoclonal antibodies in their mechanism of action. It has been demonstrated that radiation of the mediastinum as a result of treatment for early stage breast cancer or Hodgkin's lymphoma is associated with late cardiac repercussions [185]. For instance, a surgical and autopsy study of radiation-induced heart disease found valve injuries in $70 \%$ (12 out of 17 available) of studied hearts, although only eight of these were diagnosed with clinically significant dysfunction. Similarly, 63\% of myocardiums (10 out of 16 available) exhibited fibrosis related to radiation [186]. The main mechanisms associated with these phenomena are inflammation and vascular damage, which lead to cellular death [187]. This can be related to heart fibrosis found in the aforementioned study.

In the case of breast cancer, a case-control study that evaluated women with major coronary events after oncotherapy found that incidental exposure of the heart increased the rate of major coronary events in $7.4 \%$ per gray of radiation [188], establishing a direct correlation between the dose of radiotherapy and the risk of developing heart conditions. On the other hand, Hodgkin's lymphoma patients, in an echography study, were found to present Grade 1+ (scale: 0-3) aortic and/or mitral valvar regurgitation in $24 \%$ of the patients ( $15 \%$ aortic, $7 \%$ mitral, and $2 \%$ both) [189].

\section{Strain scores in echocardiography}

Strain is a term used in echocardiography to describe local shortening, thickening, and lengthening of the myocardium as a measure of regional LV function. It can be measured by tissue Doppler imaging (TDI) or by speckle-tracking echocardiography (STE), the latter being the most widely used strain modality.

Monitoring cardiac function and administration of appropriate therapy during chemotherapy is essential in current clinical practice. Nonetheless, no high-level evidence exists to guide the choice of imaging method or frequency of measurements. LVEF should be measured prior to chemotherapy using echocardiography. Patients who develop heart failure during chemotherapy are treated with standard guideline-based heart failure therapy just as any other heart failure patient [190]. For patients who develop asymptomatic LV dysfunction, however, there is not sufficient evidence to give firm recommendations with regard to medical therapy [191]. 
When a reduction in LVEF during chemotherapy is established, it may be too late for treatment [192]. Reduction in myocardial strain precedes significant change in LVEF [193]. A relative decrease in global longitudinal strain (GLS) $>15 \%$ compared with the baseline is likely to be of clinical significance, whereas a decrease $<8 \%$ is not [194]. Although strain imaging may detect subclinical myocardial changes, the value of these changes in predicting a clinical outcome is still unknown. A combination of strain imaging with ultrasensitive troponin has been proposed [191].

\section{Conclusions}

Ongoing efforts are needed to provide a better understanding of the frequency, mechanisms of disease, prevention, and treatment of cardiovascular complications induced by the newer, novel chemotherapeutic agents. Development of a cardio-oncology discipline is warranted, in order to promote task forces aiming at the creation of oncology patient-centered guidelines for the detection, prevention, and treatment of potential cardiovascular side effects associated with newer cancer therapies.

\section{Additional Information \\ Disclosures}

Conflicts of interest: In compliance with the ICMJE uniform disclosure form, all authors declare the following: Payment/services info: All authors have declared that no financial support was received from any organization for the submitted work. Financial relationships: All authors have declared that they have no financial relationships at present or within the previous three years with any organizations that might have an interest in the submitted work. Other relationships: All authors have declared that there are no other relationships or activities that could appear to have influenced the submitted work.

\section{Acknowledgements}

We would like to thank Eng. Patricio Pasco for his invaluable help with digitalization of the figures presented in this Review Article.

\section{References}

1. Schlessinger J: Cell signaling by receptor tyrosine kinases . Cell. 2000, 103:211-25. 10.1016/S00928674(00)00114-8

2. van der Geer P, Hunter T, Lindberg RA: Receptor protein-tyrosine kinases and their signal transduction pathways. Annu Rev Cell Biol. 1994, 10:251-337. 10.1146/annurev.cb.10.110194.001343

3. Krause DS, Van Etten RA: Tyrosine kinases as targets for cancer therapy . N Engl J Med. 2005, 353:172-87. 10.1056/NEJMra044389

4. Druker BJ, Sawyers CL, Kantarjian H, et al.: Activity of a specific inhibitor of the BCR-ABL tyrosine kinase in the blast crisis of chronic myeloid leukemia and acute lymphoblastic leukemia with the Philadelphia chromosome. N Engl J Med. 2001, 344:1038-42. 10.1056/NEJM200104053441402

5. Force T, Krause DS, Van Etten RA: Molecular mechanisms of cardiotoxicity of tyrosine kinase inhibition. Nat Rev Cancer. 2007, 7:332-44. 10.1038/nrc2106

6. Yeh ETH, Bickford CL: Cardiovascular complications of cancer therapy. J Am Coll Cardiol. 2009, 53:2231-47. 10.1016/j.jacc.2009.02.050

7. Perik P, Rikhof B, De Jong F, et al.: Results of plasma N-terminal pro B-type natriuretic peptide and cardiac troponin monitoring in GIST patients do not support the existence of imatinibinduced cardiotoxicity. Ann Oncol. 2008, 19:359-61. 10.1093/annonc/mdm468

8. Kerkelä R, Grazette L, Yacobi R, et al.: Cardiotoxicity of the cancer therapeutic agent imatinib mesylate. Nat Med. 2006, 12:908-16. 10.1038/nm1446

9. Talpaz M, Shah NP, Kantarjian H, et al.: Dasatinib in imatinib-resistant Philadelphia chromosome-positive leukemias. N Engl J Med. 2006, 354:2531-41. 10.1056/NEJMoa055229

10. Highlights of Prescribing Information - Sprycel (Desatinib). (2006). Accessed: July 16, 2016: http://www.accessdata.fda.gov/drugsatfda_docs/label/2010/021986s7s8lbl.pdf.

11. Kantarjian HM, Giles F, Gattermann N, et al.: Nilotinib (formerly AMN107), a highly selective 
BCR-ABL tyrosine kinase inhibitor, is effective in patients with Philadelphia chromosomepositive chronic myelogenous leukemia in chronic phase following imatinib resistance and intolerance. Blood. 2007, 110:3540-46. 10.1182/blood-2007-03-080689

12. Lu Z, Wu C-YC, Jiang Y-P, et al.: Suppression of phosphoinositide 3-kinase signaling and alteration of multiple ion currents in drug-induced long QT syndrome. Sci Transl Med. 2012, 4:131ra50. 10.1126/scitranslmed.3003623

13. Hynes NE, Lane HA: ERBB receptors and cancer: the complexity of targeted inhibitors . Nat Rev Cancer. 2005, 5:341-54. 10.1038/nrc1609

14. Junttila TT, Akita RW, Parsons K, et al.: Ligand-independent HER2/HER3/PI3K complex is disrupted by trastuzumab and is effectively inhibited by the PI3K inhibitor GDC-0941. Cancer Cell. 2009, 15:429-40. 10.1016/j.ccr.2009.03.020

15. Geyer CE, Forster J, Lindquist D, et al.: Lapatinib plus capecitabine for HER2-positive advanced breast cancer. N Engl J Med. 2006, 355:2733-43. 10.1056/NEJMoa064320

16. Perez EA, Koehler M, Byrne J, et al.: Cardiac safety of lapatinib: pooled analysis of 3689 patients enrolled in clinical trials in Mayo Clinic Proceedings. Mayo Clin Proc. 2008, 83:679-86.

10.4065/83.6.679

17. Kloth J, Pagani A, Verboom M, Malovini A, Napolitano C, Kruit W, Sleijfer S, Steeghs N, Zambelli A, Mathijssen R: Incidence and relevance of QTc-interval prolongation caused by tyrosine kinase inhibitors. Br J Cancer. 2015, 112(6):1011-1016. 10.1038/bjc.2015.82

18. Ghatalia P, Je Y, Kaymakcalan M, et al.: QTc interval prolongation with vascular endothelial growth factor receptor tyrosine kinase inhibitors. Br J Cancer. 2015, 112:296-305.

10.1038/bjc.2014.564

19. Senderowicz AM, Johnson JR, Sridhara R, et al.: Erlotinib/gemcitabine for first-line treatment of locally advanced or metastatic adenocarcinoma of the pancreas. Oncology (Williston Park). 2007, 21:1696-706.

20. Burotto M, Manasanch EE, Wilkerson J, Fojo T: Gefitinib and erlotinib in metastatic non-small cell lung cancer: a meta-analysis of toxicity and efficacy of randomized clinical trials. Oncologist. 2015, 20:400-10. 10.1634/theoncologist.2014-0154

21. Lynch TJ, Bell DW, Sordella R, et al.: Activating mutations in the epidermal growth factor receptor underlying responsiveness of non-small-cell lung cancer to gefitinib. N Engl J Med. 2004, 350:2129-39. 10.1056/NEJMoa040938

22. Paez JG, Jänne PA, Lee JC, et al.: EGFR mutations in lung cancer: correlation with clinical response to gefitinib therapy. Science. 2004, 304:1497-500. 10.1126/science.1099314

23. Pao W, Miller V, Zakowski M, et al.: EGF receptor gene mutations are common in lung cancers from "never smokers" and are associated with sensitivity of tumors to gefitinib and erlotinib. Proc Natl Acad Sci U S A. 2004, 101:13306-11. 10.1073/pnas.0405220101

24. Petrelli F, Cabiddu M, Borgonovo K, Barni S: Risk of venous and arterial thromboembolic events associated with anti-EGFR agents: a meta-analysis of randomized clinical trials. Ann Oncol. 2012, 23:1672-79. 10.1093/annonc/mdr592

25. Moore RA, Adel N, Riedel E, et al.: High incidence of thromboembolic events in patients treated with cisplatin-based chemotherapy: a large retrospective analysis. J Clin Oncol. 2011, 29:2011-35. 10.1200/JCO.2011.35.5669

26. Zecchina G, Ghio P, Bosio S, et al.: Reactive thrombocytosis might contribute to chemotherapyrelated thrombophilia in patients with lung cancer. Clin Lung Cancer. 2007, 8:264-67. 10.3816/CLC.2007.n.004

27. Mendel DB, Laird AD, Xin X, et al.: In vivo antitumor activity of SU11248, a novel tyrosine kinase inhibitor targeting vascular endothelial growth factor and platelet-derived growth factor receptors determination of a pharmacokinetic/pharmacodynamic relationship. Clin Cancer Res. 2003, 9:327-37.

28. Le Tourneau C, Raymond E, Faivre S: Sunitinib: a novel tyrosine kinase inhibitor. A brief review of its therapeutic potential in the treatment of renal carcinoma and gastrointestinal stromal tumors (GIST). Ther Clin Risk Manag. 2007, 3:341-48.

29. Demetri GD, van Oosterom AT, Garrett CR, et al.: Efficacy and safety of sunitinib in patients with advanced gastrointestinal stromal tumour after failure of imatinib: a randomised controlled trial. Lancet. 2006, 368:1329-38. 10.1016/S0140-6736(06)69446-4

30. Chu TF, Rupnick MA, Kerkela R, et al.: Cardiotoxicity associated with tyrosine kinase inhibitor sunitinib. Lancet. 2007, 370:2011-19. 10.1016/S0140-6736(07)61865-0

31. Khakoo AY, Kassiotis CM, Tannir N, et al.: Heart failure associated with sunitinib malate. Cancer. 2008, 112:2500-8. 10.1002/cncr.23460

32. Chintalgattu V, Ai D, Langley RR, et al.: Cardiomyocyte PDGFR- $\beta$ signaling is an essential 
component of the mouse cardiac response to load-induced stress. J Clin Invest. 2010, 120:472-84. 10.1172/JCI39434

33. Vaklavas C, Lenihan D, Kurzrock R, Tsimberidou AM: Anti-vascular endothelial growth factor therapies and cardiovascular toxicity: what are the important clinical markers to target?.

Oncologist. 2010, 15:130-41. 10.1634/theoncologist.2009-0252

34. Baliga RR, Givertz M, Pitt B: Management of Heart Failure: Volume 1: Medical. Baliga RR, Givertz M, Pitt B (ed): Springer-Verlag, London; 2008. 10.1007/978-1-84800-102-2

35. Telli M, Witteles R, Fisher G, Srinivas S: Cardiotoxicity associated with the cancer therapeutic agent sunitinib malate. Ann Oncol. 2008, 19:1613-18. 10.1093/annonc/mdn168

36. León-Mateos L, Mosquera J, Antón Aparicio L: Treatment of sunitinib-induced hypertension in solid tumor by nitric oxide donors. Redox Biol. 2015, 6:421-25. 10.1016/j.redox.2015.09.007

37. Wilhelm SM, Carter C, Tang L, et al.: BAY 43-9006 exhibits broad spectrum oral antitumor activity and targets the RAF/MEK/ERK pathway and receptor tyrosine kinases involved in tumor progression and angiogenesis. Cancer Res. 2004, 64:7099-109. 10.1158/0008-5472.CAN-04-1443

38. Guevremont C, Jeldres C, Perrotte P, Karakiewicz PI: Sorafenib in the management of metastatic renal cell carcinoma. Curr Oncol. 2009, 16:S27-32. 10.3747/co.v16i0.430

39. Sablin M, Bouaita L, Balleyguier C, et al.: Sequential use of sorafenib and sunitinib in renal cancer: Retrospective analysis in 90 patients. J Clin Oncol. 2007, 25:5038.

40. Kane RC, Farrell AT, Saber H, et al.: Sorafenib for the treatment of advanced renal cell carcinoma . Clin Cancer Res. 2006, 12:7271-78. 10.1158/1078-0432.CCR-06-1249

41. Gridelli C, Maione P, Del Gaizo F, et al.: Sorafenib and sunitinib in the treatment of advanced non-small cell lung cancer. Oncologist. 2007, 12:191-200. 10.1634/theoncologist.12-2-191

42. Mego M, Reckova M, Obertova J, et al.: Increased cardiotoxicity of sorafenib in sunitinibpretreated patients with metastatic renal cell carcinoma. Ann Oncol. 2007, 18:1906-7. 10.1093/annonc/mdm489

43. Schmidinger M, Zielinski CC, Vogl UM, et al.: Cardiac toxicity of sunitinib and sorafenib in patients with metastatic renal cell carcinoma. J Clin Oncol. 2008, 26:5204-12. 10.1200/JCO.2007.15.6331

44. Fujio Y, Nguyen T, Wencker D, et al.: Akt promotes survival of cardiomyocytes in vitro and protects against ischemia-reperfusion injury in mouse heart. Circulation. 2000, 101:660-67. 10.1161/01.CIR.101.6.660

45. Cook SA, Matsui T, Li L, Rosenzweig A: Transcriptional effects of chronic Akt activation in the heart. J Biol Chem. 2002, 277:22528-33. 10.1074/jbc.M201462200

46. Klein M, Schermuly RT, Ellinghaus P, et al.: Combined tyrosine and serine/threonine kinase inhibition by sorafenib prevents progression of experimental pulmonary hypertension and myocardial remodeling. Circulation. 2008, 118:2081-90. 10.1161/CIRCULATIONAHA.108.779751

47. Muslin AJ: Role of raf proteins in cardiac hypertrophy and cardiomyocyte survival. . Trends Cardiovasc Med. 2005, 15:225-229. 10.1016/j.tcm.2005.06.008

48. Will Y, Dykens JA, Nadanaciva S, et al.: Effect of the multitargeted tyrosine kinase inhibitors imatinib, dasatinib, sunitinib, and sorafenib on mitochondrial function in isolated rat heart mitochondria and H9c2 cells. Toxicol Sci. 2008, 106:153-61. 10.1093/toxsci/kfn157

49. Yamaguchi O, Watanabe T, Nishida K, et al.: Cardiac-specific disruption of the c-raf-1 gene induces cardiac dysfunction and apoptosis. J Clin Invest. 2004, 114:937-43. 10.1172/JCI20317

50. Wu S, Chen JJ, Kudelka A, et al.: Incidence and risk of hypertension with sorafenib in patients with cancer: a systematic review and meta-analysis. Lancet Oncol. 2008, 9:117-23. 10.1016/S14702045(08)70003-2

51. Veronese ML, Mosenkis A, Flaherty KT, et al.: Mechanisms of hypertension associated with BAY 43-9006. J Clin Oncol. 2006, 24:1363-69. 10.1200/JCO.2005.02.0503

52. Ryan CW, Goldman BH, Lara PN, et al.: Sorafenib with interferon alfa-2b as first-line treatment of advanced renal carcinoma: a phase II study of the Southwest Oncology Group. J Clin Oncol. 2007, 25:3296-301. 10.1200/JCO.2007.11.1047

53. Zhang X, Shao Y, Wang K: Incidence and risk of hypertension associated with cabozantinib in cancer patients: a systematic review and meta-analysis. Expert Rev Clin Pharmacol. 2016, 9:110915. 10.1080/17512433.2016.1190269

54. Center for Drug Evaluation and Research. Application number: 203756Orig1s000. Risk Assessment and Risk Mitigation Review(s). (2012). Accessed: July 15th 2016: http://www.accessdata.fda.gov/drugsatfda_docs/nda/2012/203756Orig1s000RiskR.pdf.

55. Center for Drug Evaluation and Research. Application number: 203756Orig1s000. Cross Discipline Team Leader Review. (2012). Accessed: July 15th 2016: http://www.accessdata.fda.gov/drugsatfda_docs/nda/2012/203756Orig1s000CrossR.pdf. 
56. Clerk A, Connell L, Pipe M, et al.: P95: Effects of dabrafenib and trametinib, cancer therapies that target BRaf and MEK, on cardiac signalling. Cardiovasc Res. 2014, 103:S16. 10.1093/cvr/cvu082.37

57. Luke JJ, Hodi FS: Ipilimumab, vemurafenib, dabrafenib, and trametinib: synergistic competitors in the clinical management of BRAF mutant malignant melanoma. Oncologist. 2013, 18:717-25. 10.1634/theoncologist.2012-0391

58. Shah RR, Morganroth J: Update on cardiovascular safety of tyrosine kinase inhibitors: with a special focus on QT interval, left ventricular dysfunction and overall risk/benefit. Drug Saf. 2015, 38:693-710. 10.1007/s40264-015-0300-1

59. Johnson DB, Flaherty KT, Weber JS, et al.: Combined BRAF (Dabrafenib) and MEK inhibition (Trametinib) in patients with BRAFV600-mutant melanoma experiencing progression with singleagent BRAF inhibitor. J Clin Oncol. 2014, 32:3697-704. 10.1200/JCO.2014.57.3535

60. Schlumberger M, Tahara M, Wirth LJ, et al.: Lenvatinib versus placebo in radioiodine-refractory thyroid cancer. N Engl J Med. 2015, 372:621-30. 10.1056/NEJMoa1406470

61. Nair A, Lemery SJ, Yang J, et al.: FDA approval summary: Lenvatinib for progressive, radio-iodinerefractory differentiated thyroid cancer. Clin Cancer Res. 2015, 21:5205-208. 10.1158/10780432.CCR-15-1377

62. Smith J: The cardiotoxicity of cancer-related drug therapies . US Pharm. 2014, 39:HS2-HS10.

63. Sanford D, Kantarjian H, Skinner J, et al.: Phase II trial of ponatinib in patients with chronic myeloid leukemia resistant to one previous tyrosine kinase inhibitor. Haematologica. 2015, 100:e494-e495. 10.3324/haematol.2015.132845

64. Wehrle J, Pahl HL, von Bubnoff N: Ponatinib: a third-generation inhibitor for the treatment of CML. Recent Results Cancer Research. Martens UM (ed): Springer-Verlag, Berlin Heidelberg; 2014. 201:99-107. 10.1007/978-3-642-54490-3_5

65. Cortes JE, Kim DW, Pinilla-Ibarz J, et al.: A phase 2 trial of ponatinib in Philadelphia chromosome-positive leukemias. N Engl J Med. 2013, 369:1783-96. doi:10.1056/NEJMoa1306494

66. Cortes JE, Kim DW, Pinilla-Ibarz J, et al.: Long-term follow-up of ponatinib efficacy and safety in the phase 2 PACE trial. Blood. 2014, 124:3135.

67. Finnin MS, Donigian JR, Cohen A, et al.: Structures of a histone deacetylase homologue bound to the TSA and SAHA inhibitors. Nature. 1999, 401:188-93. 10.1038/43710

68. Gilbert J, Baker SD, Bowling MK, et al.: A phase I dose escalation and bioavailability study of oral sodium phenylbutyrate in patients with refractory solid tumor malignancies. Clin Cancer Res. 2001, 7:2292-300.

69. Gore SD, Weng LJ, Figg WD, et al.: Impact of prolonged infusions of the putative differentiating agent sodium phenylbutyrate on myelodysplastic syndromes and acute myeloid leukemia. Clin Cancer Res. 2002, 8:963-70.

70. Jones PA, Baylin SB: The fundamental role of epigenetic events in cancer . Nature Rev Genet. 2002, 3:415-28. 10.1038/nrg816

71. Workman J, Kingston R: Alteration of nucleosome structure as a mechanism of transcriptional regulation. Annu Rev Biochem. 1998, 67:545-79. 10.1146/annurev.biochem.67.1.545

72. Rosato RR, Grant S: Histone deacetylase inhibitors: insights into mechanisms of lethality . Expert Opin Ther Targets. 2005, 9:809-24. 10.1517/14728222.9.4.809

73. Peng X, Pentassuglia L, Sawyer DB: Emerging anticancer therapeutic targets and the cardiovascular system: is there cause for concern?. Circ Res. 2010, 106:1022-34.

10.1161/CIRCRESAHA.109.211276

74. Zhang CL, McKinsey TA, Chang S, et al.: Class II histone deacetylases act as signal-responsive repressors of cardiac hypertrophy. Cell. 2002, 110:479-88. 10.1016/\$0092-8674(02)00861-9

75. Gusterson RJ, Jazrawi E, Adcock IM, Latchman DS: The transcriptional co-activators CREB-binding protein (CBP) and p300 play a critical role in cardiac hypertrophy that is dependent on their histone acetyltransferase activity. J Biol Chem. 2003, 278:6838-47. 10.1074/jbc.M211762200

76. Kaneda R, Takada S, Yamashita Y, et al.: Genome-wide histone methylation profile for heart failure. Genes. 2009, 14:69-77. 10.1111/j.1365-2443.2008.01252.x

77. Product monograph: Xalkori. (2016). Accessed: November 2016: http://www.pfizer.ca/sites/g/files/g10028126/f/201604/XALKORI_190668_PM_DC_24March2016_E.pd

78. Olsen EA, Kim YH, Kuzel TM, et al.: Phase IIb multicenter trial of vorinostat in patients with persistent, progressive, or treatment refractory cutaneous T-cell lymphoma. J Clin Oncol. 2007, 25:3109-15. 10.1200/JCO.2006.10.2434

79. Bagnes C, Panchuk PN, Recondo G: Antineoplastic chemotherapy induced QTc prolongation. Curr Drug Saf. 2010, 5:93-96. 10.2174/157488610789869111

80. Duvic M, Talpur R, Ni X, et al.: Phase 2 trial of oral vorinostat (suberoylanilide hydroxamic acid, SAHA) for refractory cutaneous T-cell lymphoma (CTCL). Blood. 2007, 109:31-39. 10.1182/blood- 
81. Schaefer EW, Loaiza-Bonilla A, Juckett M, et al.: A phase 2 study of vorinostat in acute myeloid leukemia. Haematologica. 2009, 94:1375-82. 10.3324/haematol.2009.009217

82. Engür S, Dikmen M, Öztürk Y: Comparison of antiproliferative and apoptotic effects of a novel proteasome inhibitor MLN2238 with bortezomib on K562 chronic myeloid leukemia cells. Immunopharmacol Immunotoxicol. 2016, 38:87-97. 10.3109/08923973.2015.1122616

83. Esparís-Ogando A, Alegre A, Aguado B, et al.: Bortezomib is an efficient agent in plasma cell leukemias. Int J Cancer. 2005, 114:665-67. 10.1002/ijc.20793

84. Kitagawa K, Kotake Y, Kitagawa M: Ubiquitin-mediated control of oncogene and tumor suppressor gene products. Cancer Sci. 2009, 100:1374-81. 10.1111/j.1349-7006.2009.01196.x

85. Mearini G, Schlossarek S, Willis MS, Carrier L: The ubiquitin-proteasome system in cardiac dysfunction. Biochim Biophys Acta. 2008, 1782:749-63. 10.1016/j.bbadis.2008.06.009

86. Powell SR: The ubiquitin-proteasome system in cardiac physiology and pathology . Am J Physiol. 2006, 291:H1-H19. 10.1152/ajpheart.00062.2006

87. Hacihanefioglu A, Tarkun P, Gonullu E: Acute severe cardiac failure in a myeloma patient due to proteasome inhibitor bortezomib. Int J Hematol. 2008, 88:219-22. 10.1007/s12185-008-0139-7

88. Voortman J, Giaccone G: Severe reversible cardiac failure after bortezomib treatment combined with chemotherapy in a non-small cell lung cancer patient: a case report. BMC Cancer. 2006, 6:129. 10.1186/1471-2407-6-129

89. Orciuolo E, Buda G, Cecconi N, et al.: Unexpected cardiotoxicity in haematological bortezomib treated patients. Br J Haematol. 2007, 138:396-97. 10.1111/j.1365-2141.2007.06659.x

90. Richardson PG, Sonneveld P, Schuster MW, et al.: Bortezomib or high-dose dexamethasone for relapsed multiple myeloma. N Engl J Med. 2005, 352:2487-98. 10.1056/NEJMoa043445

91. Yang H, Zonder JA, Dou QP: Clinical development of novel proteasome inhibitors for cancer treatment. Expert Opin Investig Drugs. 2009, 18:957-971. 10.1517/13543780903002074

92. van Hees HW, Li YP, Ottenheijm CA, et al.: Proteasome inhibition improves diaphragm function in congestive heart failure rats. Am J Physiol Lung Cell Mol Physiol. 2008, 294:L1260-68. 10.1152/ajplung.00035.2008

93. Bulteau AL, Lundberg KC, Humphries KM, et al.: Oxidative modification and inactivation of the proteasome during coronary occlusion/reperfusion. J Biol Chem. 2001, 276:30057-63. 10.1074/jbc.M100142200

94. Presta LG, Chen H, O'connor SJ, et al.: Humanization of an anti-vascular endothelial growth factor monoclonal antibody for the therapy of solid tumors and other disorders. Cancer Res. 1997, 57:4593-99.

95. FDA Final labeling text: AVASTIN (Bevacizumab) . (2009). Accessed: July 2016: http://www.accessdata.fda.gov/drugsatfda_docs/label/2009/125085s0169lbl.pdf.

96. Miller KD, Chap LI, Holmes FA, et al.: Randomized phase III trial of capecitabine compared with bevacizumab plus capecitabine in patients with previously treated metastatic breast cancer. J Clin Oncol. 2005, 23:792-99. 10.1200/JCO.2005.05.098

97. Miller K, Wang M, Gralow J, et al.: Paclitaxel plus bevacizumab versus paclitaxel alone for metastatic breast cancer. N Engl J Med. 2007, 357:2666-76. 10.1056/NEJMoa072113

98. Kabbinavar FF, Schulz J, McCleod M, et al.: Addition of bevacizumab to bolus fluorouracil and leucovorin in first-line metastatic colorectal cancer: results of a randomized phase II trial. J Clin Oncol. 2005, 23:3697-705. 10.1200/JCO.2005.05.112

99. Yang JC, Haworth L, Sherry RM, et al.: A randomized trial of bevacizumab, an anti-vascular endothelial growth factor antibody, for metastatic renal cancer. N Engl J Med. 2003, 349:427-34. 10.1056/NEJMoa021491

100. Hurwitz H, Fehrenbacher L, Novotny W, et al.: Bevacizumab plus irinotecan, fluorouracil, and leucovorin for metastatic colorectal cancer. N Engl J Med. 2004, 350:2335-42. 10.1056/NEJMoa032691

101. Cobleigh MA, Langmuir VK, Sledge GW, et al.: A phase I/II dose-escalation trial of bevacizumab in previously treated metastatic breast cancer. Semin Oncol. 2003, 30:117-24. 10.1053/j.seminoncol.2003.08.013

102. Pande A, Lombardo J, Spangenthal E, Javle M: Hypertension secondary to anti-angiogenic therapy: experience with bevacizumab. Anticancer Res. 2007, 27:3465-70.

103. Johnson DH, Fehrenbacher L, Novotny WF, et al.: Randomized phase II trial comparing bevacizumab plus carboplatin and paclitaxel with carboplatin and paclitaxel alone in previously untreated locally advanced or metastatic non-small-cell lung cancer. J Clin Oncol. 2004, 22:218491. 10.1200/JCO.2004.11.022

104. Siegel AB, Cohen EI, Ocean A, et al.: Phase II trial evaluating the clinical and biologic effects of 
bevacizumab in unresectable hepatocellular carcinoma. J Clin Oncol. 2008, 26:2992-98. 10.1200/JCO.2007.15.9947

105. Burger RA, Sill MW, Monk BJ, et al.: Phase II trial of bevacizumab in persistent or recurrent epithelial ovarian cancer or primary peritoneal cancer: a Gynecologic Oncology Group Study. J Clin Oncol. 2007, 25:5165-71. 10.1200/JCO.2007.11.5345

106. Fiedler W, Serve H, Döhner H, et al.: A phase 1 study of SU11248 in the treatment of patients with refractory or resistant acute myeloid leukemia (AML) or not amenable to conventional therapy for the disease. Blood. 2005, 105:986-93. 10.1182/blood-2004-05-1846

107. Schneider BP, Wang M, Radovich M, et al.: Association of vascular endothelial growth factor and vascular endothelial growth factor receptor-2 genetic polymorphisms with outcome in a trial of paclitaxel compared with paclitaxel plus bevacizumab in advanced breast cancer: ECOG 2100. J Clin Oncol. 2008, 26:4672-78. 10.1200/JCO.2008.16.1612

108. Gordon M, Margolin K, Talpaz M, et al.: Phase I safety and pharmacokinetic study of recombinant human anti-vascular endothelial growth factor in patients with advanced cancer. J Clin Oncol. 2001, 19:843-50. 10.1200/JCO.2001.19.3.843

109. Azad NS, Posadas EM, Kwitkowski VE, et al.: Combination targeted therapy with sorafenib and bevacizumab results in enhanced toxicity and antitumor activity. J Clin Oncol. 2008, 26:3709-14. 10.1200/JCO.2007.10.8332

110. D'Adamo DR, Anderson SE, Albritton K, et al.: Phase II study of doxorubicin and bevacizumab for patients with metastatic soft-tissue sarcomas. J Clin Oncol. 2005, 23:7135-42. 10.1200/JCO.2005.16.139

111. Karp JE, Gojo I, Pili R, et al.: Targeting vascular endothelial growth factor for relapsed and refractory adult acute myelogenous leukemias: therapy with sequential 1-beta-darabinofuranosylcytosine, mitoxantrone, and bevacizumab. Clin Cancer Res. 2004, 10:3577-3585. 10.1158/1078-0432.CCR-03-0627

112. Noon JP, Walker BR, Webb DJ, et al.: Impaired microvascular dilatation and capillary rarefaction in young adults with a predisposition to high blood pressure. J Clin Invest. 1997, 99:1873-79. 10.1172/JCI119354

113. Felmeden DC, Spencer CG, Belgore FM, et al.: Endothelial damage and angiogenesis in hypertensive patients: relationship to cardiovascular risk factors and risk factor management. Am J Hypertens. 2003, 16:11-20. 10.1016/S0895-7061(02)03149-7

114. Kamba T, McDonald D: Mechanisms of adverse effects of anti-VEGF therapy for cancer . Brit J Cancer. 2007, 96:1788-95. 10.1038/sj.bjc.6603813

115. Friehs I, Barillas R, Vasilyev NV, et al.: Vascular endothelial growth factor prevents apoptosis and preserves contractile function in hypertrophied infant heart. Circulation. 2006, 114:1290-95. 10.1161/CIRCULATIONAHA.105.001289

116. Zentilin L, Puligadda U, Lionetti V, et al.: Cardiomyocyte VEGFR-1 activation by VEGF-B induces compensatory hypertrophy and preserves cardiac function after myocardial infarction. FASEB J. 2010, 24:1467-78. 10.1096/fj.09-143180

117. Shiojima I, Sato K, Izumiya Y, et al.: Disruption of coordinated cardiac hypertrophy and angiogenesis contributes to the transition to heart failure. J Clin Invest. 2005, 115:2108-18. 10.1172/JCI24682

118. Nalluri SR, Chu D, Keresztes R, et al.: Risk of venous thromboembolism with the angiogenesis inhibitor bevacizumab in cancer patients: a meta-analysis. JAMA. 2008, 300:2277-85. 10.1001/jama.2008.656

119. Scappaticci FA, Skillings JR, Holden SN, et al.: Arterial thromboembolic events in patients with metastatic carcinoma treated with chemotherapy and bevacizumab. J Natl Cancer Inst. 2007, 99:1232-39. 10.1093/jnci/djm086

120. Sugrue M, Yi J, Purdie D, et al.: Serious arterial thromboembolic events (sATE) in patients (pts) with metastatic colorectal cancer (mCRC) treated with bevacizumab (BV): results from the BRiTE registry. J Clin Oncol. 2007, 25:4136.

121. Nachman RL, Rafii S: Platelets, petechiae, and preservation of the vascular wall . N Engl J Med. 2008, 359:1261-70. 10.1056/NEJMra0800887

122. Slamon DJ, Godolphin W, Jones LA, et al.: Studies of the HER-2/neu proto-oncogene in human breast and ovarian cancer. Science. 1989, 244:707-12. 10.1126/science.2470152

123. Press MF, Pike MC, Chazin VR, et al.: Her-2/neu expression in node-negative breast cancer: direct tissue quantitation by computerized image analysis and association of overexpression with increased risk of recurrent disease. Cancer Res. 1993, 53:4960-70.

124. Coussens L, Yang-Feng TL, Liao YC, et al.: Tyrosine kinase receptor with extensive homology to EGF receptor shares chromosomal location with neu oncogene. Science. 1985, 230:1132-39. 


\subsection{6/science.2999974}

125. Hudziak RM, Lewis GD, Winget M, et al.: p185HER2 monoclonal antibody has antiproliferative effects in vitro and sensitizes human breast tumor cells to tumor necrosis factor. Mol Cell Biol. 1989, 9:1165-72. 10.1128/MCB.9.3.1165

126. Geiger S, Lange V, Suhl P, et al.: Anticancer therapy induced cardiotoxicity: review of the literature. Anticancer Drugs. 2010, 21:578-90. 10.1097/CAD.0b013e3283394624

127. Baselga J, Perez EA, Pienkowski T, Bell R: Adjuvant trastuzumab: a milestone in the treatment of HER-2-positive early breast cancer. Oncologist. 2006, 11:4-12. 10.1634/theoncologist.11-90001-4

128. Khakoo AY, Yeh ET: Therapy insight: management of cardiovascular disease in patients with cancer and cardiac complications of cancer therapy. Nat Clin Pract Oncol. 2008, 5:655-67. 10.1038/ncponc1225

129. Gille L, Nohl H: Analyses of the molecular mechanism of adriamycin-induced cardiotoxicity. Free Radic Biol Med. 1997, 23:775-82. 10.1016/S0891-5849(97)00025-7

130. Ewer MS, Vooletich MT, Durand JB, et al.: Reversibility of trastuzumab-related cardiotoxicity: new insights based on clinical course and response to medical treatment. J Clin Oncol. 2005, 23:782026. 10.1200/JCO.2005.13.300

131. Zhao YY, Sawyer DR, Baliga RR, et al.: Neuregulins promote survival and growth of cardiac myocytes Persistence of ErbB2 and ErbB4 expression in neonatal and adult ventricular myocytes. J Biol Chem. 1998, 273:10261-69. 10.1074/jbc.273.17.10261

132. De Keulenaer GW, Doggen K, Lemmens K: The vulnerability of the heart as a pluricellular paracrine organ lessons from unexpected triggers of heart failure in targeted ErbB2 anticancer therapy. Circ Res. 2010, 106:35-46. 10.1161/CIRCRESAHA.109.205906

133. Perik PJ, Vries EG, Gietema JA, et al.: Serum HER2 levels are increased in patients with chronic heart failure. Eur J Heart Fail. 2007, 9:173-77. 10.1016/j.ejheart.2006.05.010

134. Slamon DJ, Leyland-Jones B, Shak S, et al.: Use of chemotherapy plus a monoclonal antibody against HER2 for metastatic breast cancer that overexpresses HER2. N Engl J Med. 2001, 344:78392. 10.1056/NEJM200103153441101

135. Cheng H, Force T: Molecular mechanisms of cardiovascular toxicity of targeted cancer therapeutics. Circ Res. 2010, 106:21-34. 10.1161/CIRCRESAHA.109.206920

136. Bria E, Cuppone F, Fornier M, et al.: Cardiotoxicity and incidence of brain metastases after adjuvant trastuzumab for early breast cancer: the dark side of the moon? A meta-analysis of the randomized trials. Breast Cancer Res Treat. 2008, 109:231-39. 10.1007/s10549-007-9663-Z

137. Swain SM, Whaley FS, Ewer MS: Congestive heart failure in patients treated with doxorubicin . Cancer. 2003, 97:2869-79. 10.1002/cncr.11407

138. Perez EA, Rodeheffer R: Clinical cardiac tolerability of trastuzumab. J Clin Oncol. 2004, 22:322-29. 10.1200/JCO.2004.01.120

139. Janas E, Priest R, Wilde J, et al.: Rituxan (anti-CD20 antibody)-induced translocation of CD20 into lipid rafts is crucial for calcium influx and apoptosis. Clin Exp Immunol. 2005, 139:439-46. 10.1111/j.1365-2249.2005.02720.x

140. Hofmeister JK, Cooney D, Coggeshall KM: Clustered CD20 induced apoptosis: src-family kinase, the proximal regulator of tyrosine phosphorylation, calcium influx, and caspase 3-dependent apoptosis. Blood Cells Mol Dis. 2000, 26:133-43. 10.1006/bcmd.2000.0287

141. Syrigou E, Makrilia N, Koti I, et al.: Hypersensitivity reactions to antineoplastic agents: an overview. Anticancer Drugs. 2009, 20:1-6. 10.1097/CAD.0b013e32831961b3

142. FDA Final Labeling Text: Rituxan (Rituximab). (2010). Accessed: July 2016: http://www.accessdata.fda.gov/drugsatfda_docs/label/2010/103705s5311lbl.pdf.

143. Kilickap S, Yavuz B, Aksoy S, et al.: Addition of rituximab to chop does not increase the risk of cardiotoxicity in patients with non-Hodgkin's lymphoma. Medic Oncol. 2008, 25:437-42. 10.1007/s12032-008-9062-2

144. Cersosimo RJ: Monoclonal antibodies in the treatment of cancer, Part 1 . Am J Health Syst Pharm. 2003, 60:1531-48.

145. Robak T: Alemtuzumab for B-cell chronic lymphocytic leukemia. Expert Rev Anticancer Ther. 2008, 8:1033-51. 10.1586/14737140.8.7.1033

146. Lenihan DJ, Alencar AJ, Yang D, et al.: Cardiac toxicity of alemtuzumab in patients with mycosis fungoides/Sezary syndrome. Blood. 2004, 104:655-58. 10.1182/blood-2003-07-2345

147. Costa A, Zaki B, Yen S, et al.: Short course of bendamustine and rituximab followed by 90Yibritumomab tiuxetan in patients with chemotherapy-naive follicular lymphoma (FOL-BRITe): Final report of response rates and progression free survival. Blood. 2016, 128:1793.

148. Dillman RO: Radiolabeled anti-CD20 monoclonal antibodies for the treatment of B-cell lymphoma. J Clin Oncol. 2002, 20:3545-57. 10.1200/JCO.2002.02.126 
149. Horning SJ, Younes A, Jain V, et al.: Efficacy and safety of tositumomab and iodine-131 tositumomab (Bexxar) in B-cell lymphoma, progressive after rituximab. J Clin Oncol. 2005, 23:712-19. 10.1200/JCO.2005.07.040

150. Jacene H, Crandall J, Kasamon Y, et al.: Initial experience with tositumomab and I-131-labeled tositumomab for treatment of relapsed/refractory Hodgkin lymphoma. Mol Imaging Biol. 2017, 19:429-36. 10.1007/s11307-016-1019-9

151. Burtness B: Cetuximab and cisplatin for chemotherapy-refractory squamous cell cancer of the head and neck. J Clin Oncol. 2005, 23:5440-42. 10.1200/JCO.2005.02.002

152. Zhang Y, Yang J, Ding M, et al.: Tumor-penetration and antitumor efficacy of cetuximab are enhanced by co-administered iRGD in a murine model of human NSCLC. Oncol Lett. 2016, 12:3241-49. 10.3892/ol.2016.5081

153. Vectibix ${ }^{\circledR}$ (panitumumab) - FDA: Highlights of Prescribing Information. (2009). Accessed: July 2016: http://www.accessdata.fda.gov/drugsatfda_docs/label/2009/125147s080lbl.pdf.

154. Arzerra (Ofatumumab) - FDA: Highlights of Prescribing Information . (2009). Accessed: July 2016: http://www.accessdata.fda.gov/drugsatfda_docs/label/2009/125326lbl.pdf.

155. List A, Dewald G, Bennett J, et al.: Lenalidomide in the myelodysplastic syndrome with chromosome 5q deletion. N Engl J Med. 2006, 355:1456-65. 10.1056/NEJMoa061292

156. REVLIMID (lenalidomide) capsules, for oral use - FDA: Highlights of Prescribing Information . (2013). Accessed: July 2016: http://www.accessdata.fda.gov/drugsatfda_docs/label/2013/021880s034lbl.pdf.

157. Bennett CL, Schumock GT, Desai AA, et al.: Thalidomide-associated deep vein thrombosis and pulmonary embolism. Am J Med. 2002, 113:603-606. 10.1016/S0002-9343(02)01300-1

158. Dimopoulos M, Spencer A, Attal M, et al.: Lenalidomide plus dexamethasone for relapsed or refractory multiple myeloma. N Engl J Med. 2007, 357:2123-32. 10.1056/NEJMoa070594

159. Robert C, Schachter J, Long GV, et al.: Pembrolizumab versus ipilimumab in advanced melanoma . N Engl J Med. 2015, 372:2521-32. 10.1056/NEJMoa1503093

160. Mallarkey G, Mangoni AA: Targeting precision medicine toxicity: recent developments . Ther Adv Drug Saf. 2015, 6:4-14. 10.1177/2042098614560737

161. Okazaki T, Tanaka Y, Nishio R, et al.: Autoantibodies against cardiac troponin I are responsible for dilated cardiomyopathy in PD-1-deficient mice. Nat Med. 2003, 9:1477-83. 10.1038/nm955

162. FDA - Rapamune (sirolimus): Highlights of Prescribing Information . (2010). Accessed: July 2016: http://www.accessdata.fda.gov/drugsatfda_docs/label/2010/021110s058lbl.pdf.

163. Kelly PA, Gruber SA, Behbod F, Kahan BD: Sirolimus, a new, potent immunosuppressive agent. Pharmacotherapy. 1997, 17:1148-56.

164. Song X, Kusakari Y, Xiao CY, et al.: mTOR attenuates the inflammatory response in cardiomyocytes and prevents cardiac dysfunction in pathological hypertrophy. Am J Physiol Cell Physiol. 2010, 299:C1256-66. 10.1152/ajpcell.00338.2010

165. Davis PJ, Davis FB, Lin H-Y: Roles and controls of mTOR in the heart. Focus on "mTOR attenuates the inflammatory response in cardiomyocytes and prevents cardiac dysfunction in pathological hypertrophy”. Am J Physiol Cell Physiol. 2010, 299:C1250-52.

166. Hands S, Proud C, Wyttenbach A: mTOR's role in ageing: protein synthesis or autophagy? . Aging (Albany NY). 2009, 1:586-97. 10.18632/aging.100070

167. FDA - Afinitor (everolimus): Highlights of Prescribing Information. (2012). Accessed: July 2016: https://www.accessdata.fda.gov/drugsatfda_docs/label/2012/022334s016lbl.pdf.

168. FDA - Torisel (temsirolimus): Highlights of Prescribing Information . (2015). Accessed: July 2016: http://www.accessdata.fda.gov/drugsatfda_docs/label/2015/022088s018lbl.pdf.

169. Sengupta PP, Northfelt DW, Gentile F, et al.: Trastuzumab-induced cardiotoxicity: heart failure at the crossroads. Mayo Clin Proc. 2008, 83:197-203. 10.4065/83.2.197

170. Bellenger N, Burgess M, Ray S, et al.: Comparison of left ventricular ejection fraction and volumes in heart failure by echocardiography, radionuclide ventriculography and cardiovascular magnetic resonance. Are they interchangeable?. Eur Heart J. 2000, 21:1387-96. 10.1053/euhj.2000.2011

171. Heart Failure Society of America: Executive Summary: HFSA 2006 Comprehensive Heart Failure Practice Guideline. J Card Fail. 2006, 12:10-38. 10.1016/j.cardfail.2005.12.001

172. Ewer MS, Yeh ETH: Cancer and the Heart, 2nd edition. Linda Mehta (ed): People's Medical Publishing House-USA, Shelton, CT; 2013.

173. Hunt SA, Abraham WT, Chin MH, et al.: ACC/AHA 2005 guideline update for the diagnosis and management of chronic heart failure in the adult. Circulation. 2005, 112:e154-235. 10.1161/CIRCULATIONAHA.105.167586

174. Yusuf SW, Razeghi P, Yeh ET: The diagnosis and management of cardiovascular disease in cancer patients. Curr Probl Cardiol. 2008, 33:163-96. 10.1016/j.cpcardiol.2008.01.002 
175. Rixe O, Billemont B, Izzedine H: Hypertension as a predictive factor of Sunitinib activity . Ann Oncol. 2007, 18:1117. 10.1093/annonc/mdm184

176. Khakoo AY, Sidman RL, Pasqualini R, Arap W: Does the renin-angiotensin system participate in regulation of human vasculogenesis and angiogenesis?. Cancer Res. 2008, 68:9112-15.

10.1158/0008-5472.CAN-08-0851

177. Lipshultz SE, Lipsitz SR, Sallan SE, et al.: Long-term enalapril therapy for left ventricular dysfunction in doxorubicin-treated survivors of childhood cancer. J Clin Oncol. 2002, 20:4517-22. 10.1200/JCO.2002.12.102

178. Nakamae H, Tsumura K, Terada Y, et al.: Notable effects of angiotensin II receptor blocker, valsartan, on acute cardiotoxic changes after standard chemotherapy with cyclophosphamide, doxorubicin, vincristine, and prednisolone. Cancer. 2005, 104:2492-98. 10.1002/cncr.21478

179. Kalay N, Basar E, Ozdogru I, et al.: Protective effects of carvedilol against anthracycline-induced cardiomyopathy. J Am Coll Cardiol. 2006, 48:2258-62. 10.1016/j.jacc.2006.07.052

180. Ghobrial IM, Rajkumar SV: Management of thalidomide toxicity. J Support Oncol. 2003, 1:194205.

181. Anderson JL, Adams CD, Antman EM, et al.: ACC/AHA 2007 guidelines for the management of patients with unstable angina/non-ST-elevation myocardial infarction: A report of the American College of Cardiology/American Heart Association Task Force on practice guidelines (Writing Committee to revise the 2002 Guidelines for the Management of Patients with Unstable Angina/Non-ST-Elevation Myocardial Infarction) developed in collaboration with the American College of Emergency Physicians, the Society for Cardiovascular Angiography and Interventions, and the Society of Thoracic Surgeons endorsed by the American Association of Cardiovascular and Pulmonary Rehabilitation and the Society for Academic Emergency Medicine. J Am Coll Cardiol. 2007, 50:e1-e157. 10.1016/j.jacc.2007.02.013

182. Antman EM, Hand M, Armstrong PW, et al.: 2007 focused update of the ACC/AHA 2004 Guidelines for the Management of Patients with ST-Elevation Myocardial Infarction: a report of the American College of Cardiology/American Heart Association Task Force on Practice Guidelines: developed in collaboration with the Canadian Cardiovascular Society endorsed by the American Academy of Family Physicians: 2007 Writing Group to review new evidence and update the ACC/AHA 2004 Guidelines for the Management of Patients With ST-Elevation Myocardial Infarction, writing on behalf of the 2004 Writing Committee. Circulation. 2008, 117:296-329. 10.1161/CIRCULATIONAHA.107.188209

183. Palumbo A, Rajkumar SV, Dimopoulos M, Richardson PG: Prevention of thalidomide-and lenalidomide-associated thrombosis in myeloma. Leukemia. 2008, 22:414-23.

10.1038/sj.leu.2405062

184. Kearon C, Kahn SR, Agnelli G, et al.: Antithrombotic therapy for venous thromboembolic disease: American College of Chest Physicians evidence-based clinical practice guidelines. Chest. 2008, 133:454S-545S. 10.1378/chest.08-0658

185. Cardiotoxicity of radiation therapy for breast cancer and other malignancies . (2017). Accessed: April 16, 2017: http://www.uptodate.com/contents/cardiotoxicity-of-radiation-therapy-forbreast-cancer-and-other-malignancies.

186. Veinot JP, Edwards WD: Pathology of radiation-induced heart disease: A surgical and autopsy study of 27 cases. Hum Pathol. 1996, 27:766-73. 10.1016/S0046-8177(96)90447-5

187. Campia U, Barac A: Cardiovascular side effects of cancer treatments . Pharm J. 2016, 8:Accessed: April 16, 2017: http://www.pharmaceutical-journal.com/research/review-article/cardiovascularside-effects-of-cancer-treatments/202016.... 10.1211/CP.2016.20201651

188. Darby SC, Ewertz M, McGale P, Bennet AM, Blom-Goldman U, Brønnum D, et al.: Risk of Ischemic Heart Disease in Women after Radiotherapy for Breast Cancer. N Engl J Med. 2013, 368 (11):987 998. 10.1056/NEJMoa1209825

189. Lund MB, Ihlen H, Voss BM, et al.: Increased risk of heart valve regurgitation after mediastinal radiation for Hodgkin's disease: an echocardiographic study. Heart. 1996, 75:591-95. 10.1136/hrt.75.6.591

190. Eschenhagen T, Force T, Ewer MS, et al.: Cardiovascular side effects of cancer therapies: a position statement from the Heart Failure Association of the European Society of Cardiology. Eur J Heart Fail. 2011, 13:1-10. 10.1093/eurjhf/hfq213

191. Smiseth O, Torp H, Opdahl A, et al.: Myocardial strain imaging: how useful is it in clinical decision making?. Eur Heart J. 2016, 37:1196-207. 10.1093/eurheartj/ehv529

192. Cardinale D, Colombo A, Lamantia G, Colombo N, Civelli M, De Giacomi G, Rubino M, Veglia F, Fiorentini C, Cipolla CM: Anthracycline-induced cardiomyopathy: clinical relevance and response to pharmacologic therapy. J Am Coll Cardiol. 2010, 55:213-220. doi: 10.1016/j.jacc.2009.03.095 
Cureus

193. Thavendiranathan P, Poulin F, Lim KD, et al.: Use of myocardial strain imaging by echocardiography for the early detection of cardiotoxicity in patients during and after cancer chemotherapy: a systematic review. J Am Coll Cardiol. 2014, 63:2751-68. 10.1016/j.jacc.2014.01.073

194. Plana JC, Galderisi M, Barac A, et al.: Expert consensus for multimodality imaging evaluation of adult patients during and after cancer therapy: a report from the American Society of Echocardiography and the European Association of Cardiovascular Imaging. Eur Heart J Cardiovasc Imaging. 2014, 15:1063-93. 10.1093/ehjci/jeu192 